\title{
INSTABILITY OF PERIODIC TRAVELING WAVES FOR THE SYMMETRIC REGULARIZED LONG WAVE EQUATION
}

\author{
JAIME ANGULO PAVA AND \\ CARLOS ALBERTO BANQUET BRANGO
}

\begin{abstract}
We prove the linear and nonlinear instability of periodic traveling wave solutions for a generalized version of the symmetric regularized long wave (SRLW) equation. Using analytic and asymptotic perturbation theory, we establish sufficient conditions for the existence of exponentially growing solutions to the linearized problem and so the linear instability of periodic profiles is obtained. An application of this approach is made to obtain the linear/nonlinear instability of cnoidal wave solutions for the modified SRLW (mSRLW) equation. We also prove the stability of dnoidal wave solutions associated to the equation just mentioned.
\end{abstract}

\section{$\S 1$. Introduction}

In this paper we study the nonlinear stability and linear/nonlinear instability of periodic traveling waves for the general model

$$
u_{t t}-u_{x x}+(f(u))_{x t}+(\mathcal{M} u)_{t t}=0
$$

where $u=u(x, t), x, t \in \mathbb{R}$, is a real-valued function, and $\mathcal{M}$ is a differential or pseudodifferential operator in the framework of periodic functions, which is defined as a Fourier multiplier operator

$$
\widehat{\mathcal{M} u}(k)=\alpha(k) \widehat{u}(k), \quad k \in \mathbb{Z},
$$

where the symbol $\alpha$ of $\mathcal{M}$ is a measurable, locally bounded, even function on $\mathbb{R}$, satisfying the condition

$$
a_{1}|k|^{m_{1}} \leq \alpha(k) \leq a_{2}(1+|k|)^{m_{2}},
$$

Received March 28, 2012. Revised January 30, 2014. Accepted August 14, 2014.

2010 Mathematics Subject Classification. Primary 35Q51; Secondary 35B35, 35B10, $35 \mathrm{C} 07$. 
for $1 \leq m_{1} \leq m_{2},|k| \geq k_{0}, \alpha(k)>b$, for all $k \in \mathbb{Z}, a_{1}, a_{2}>0$ and $f$ being a sufficient smooth function which prescribes nonlinearity. In our applications we are interested in nonlinearities of the form $f(u)=u^{p+1}$, with $p \geq 1$ a positive integer. If we consider $\mathcal{M}=-\partial_{x}^{2}$ and $p=1$, we obtain the symmetric regularized long wave (SRLW) equation which is a model for the weakly nonlinear ion acoustic and space-charge waves. The SRLW equation was introduced by Seyler and Fenstermacher [20], where a weakly nonlinear analysis of the cold electron fluid equation is made. The SRLW equation is also a model for water waves. In fact, the so-called bad Boussinesq

$$
u_{t t}-u_{x x}-\left(u^{2}\right)_{x x}-u_{x x x x}=0
$$

can be derived as a model for unidirectional water waves (see Boussinesq [8]). The equation (1.1) in the particular case $\mathcal{M}=-\partial_{x}^{2}$ and $f(u)=u^{2}$ can be obtained from (1.2) by substituting $-\partial_{x}$ for $\partial_{t}$ in the last two terms. This substitution is consistent with the assumptions made in deriving (1.2) as a model equation for water waves. So, equation (1.1) can be seen as a generalized version of the SRLW equation (henceforth, SRLW-type equation).

We are interested in the vectorial form of equation (1.1), namely,

$$
\left\{\begin{array}{l}
u_{t}+(\mathcal{M} u)_{t}+(f(u))_{x}-v_{x}=0 \\
v_{t}-u_{x}=0
\end{array}\right.
$$

For equation (1.3) we desire to find solutions of the form

$$
\left(u_{c}(x, t), v_{c}(x, t)\right)=\left(\phi_{c}(x-c t), \psi_{c}(x-c t)\right),
$$

with profiles $\phi_{c}, \psi_{c}: \mathbb{R} \rightarrow \mathbb{R}$ being $L$-periodic smooth functions and $c \in \mathbb{R}$ the wave speed. If we substitute (1.4) in (1.3) after an integration, the following pseudodifferential system is obtained:

$$
\left\{\begin{array}{l}
c \mathcal{M} \phi_{c}+c \phi_{c}-f\left(\phi_{c}\right)+\psi_{c}=A_{\phi_{c}, \psi_{c}}, \\
c \psi_{c}+\phi_{c}=B_{\phi_{c}, \psi_{c}}
\end{array}\right.
$$

where $A_{\phi_{c}, \psi_{c}}$ and $B_{\phi_{c}, \psi_{c}}$ are constants, which are considered zero in our theory.

Some symmetric considerations deserve to be mentioned before talking about instability. Since equation (1.3) is invariant under translations (if $(u(x, t), v(x, t))$ is a solution for $(1.3)$, then $(u(x+y, t), v(x+y, t))$ is also a 
solution for every $y \in \mathbb{R}$ ), we obtain that the 1-parameter group of unitary operators $\{T(y)\}_{y \in \mathbb{R}}$ defined by $T(y) f(\cdot)=f(\cdot+y)$ determines the $\left(\phi_{c}, \psi_{c}\right)$ orbit

$$
\Omega_{\left(\phi_{c}, \psi_{c}\right)}:=\left\{\left(T(y) \phi_{c}, T(y) \psi_{c}\right) ; y \in \mathbb{R}\right\} .
$$

Then, we say that $\Omega_{\left(\phi_{c}, \psi_{c}\right)}$ is stable in the periodic space $X=H_{\mathrm{per}}^{\frac{m_{2}}{2}}([0, L]) \times$ $L_{\text {per }}^{2}([0, L])$ by the flow of equation $(1.3)$, if for all $\varepsilon>0$ there is $\delta>0$ such that if $\left\|\left(u_{0}, v_{0}\right)-\left(\phi_{c}, \psi_{c}\right)\right\|_{X}<\delta$ and $(u(t), v(t))$ is the global solution of (1.3) with initial data $(u(x, 0), v(x, 0))=\left(u_{0}(x), v_{0}(x)\right)$, then

$$
\inf _{y \in \mathbb{R}}\left\|(u(t), v(t))-\left(T(y) \phi_{c}, T(y) \psi_{c}\right)\right\|_{X}<\varepsilon, \quad \forall t \in \mathbb{R} .
$$

Otherwise, the $\left(\phi_{c}, \psi_{c}\right)$-orbit is said to be orbitally unstable in $X$.

The instability behavior would happen if the solutions ceased to exist for a class of initial data close to $\left(\phi_{c}, \psi_{c}\right)$ after a finite time (blow-up case). This kind of behavior for models of dispersive type is in general a very difficult task to be addressed. In our instability study, the solutions will exist globally in time.

Consider $w(x, t)=u(x+c t, t)-\phi_{c}(x)$ and $z(x, t)=v(x+c t, t)-\psi_{c}(x)$ in (1.3); then we obtain via Taylor's theorem the next system

$$
\left\{\begin{array}{l}
\left(\partial_{t}-c \partial_{x}\right)(w+\mathcal{M} w)+\partial_{x}\left(-z+w f^{\prime}\left(\phi_{c}\right)\right)+O\left(\|(w, z)\|^{2}\right)=0 \\
\left(\partial_{t}-c \partial_{x}\right) z-w_{x}+O\left(\|(w, z)\|^{2}\right)=0
\end{array}\right.
$$

The following system,

$$
\left\{\begin{array}{l}
\left(\partial_{t}-c \partial_{x}\right)(w+\mathcal{M} w)+\partial_{x}\left(-z+w f^{\prime}\left(\phi_{c}\right)\right)=0 \\
\left(\partial_{t}-c \partial_{x}\right) z-w_{x}=0
\end{array}\right.
$$

represents the linearization of (1.3) around $\left(\phi_{c}, \psi_{c}\right)$. Our objective will be to give sufficient conditions to obtain that the solution $(w, z) \equiv(0,0)$ is unstable by the linear flow of (1.6). More exactly, we are interested in finding a growing mode solution for (1.6) of the form $\left(e^{\lambda t} u(x), e^{\lambda t} v(x)\right)$ with $\operatorname{Re} \lambda>0$. Therefore, $(u, v)$ satisfies the nonlocal differential equation

$$
\left\{\begin{array}{l}
u+\mathcal{M} u+\frac{\partial_{x}}{\lambda-c \partial_{x}}\left(-v+u f^{\prime}\left(\phi_{c}\right)\right)=0, \\
v=\frac{\partial_{x}}{\lambda-c \partial_{x}} u
\end{array}\right.
$$

where the expression $\frac{\partial_{x}}{\lambda-c \partial_{x}}$, with $\operatorname{Re} \lambda>0$, is a notation for the linear operator $\partial_{x}\left(\lambda-c \partial_{x}\right)^{-1}$. From equation (1.7) it is clear that $u$ and $v$ have zero 
mean. By using the formula for $v$ in the second equation in (1.7) and replacing it on the first equation in 1.7 , we arrive at

$$
u+\mathcal{M} u+\frac{\partial_{x}}{\lambda-c \partial_{x}}\left(f^{\prime}(\phi) u-\frac{\partial_{x}}{\lambda-c \partial_{x}} u\right)=0 .
$$

Next we consider the space of zero mean $\mathbb{V}$, more exactly,

$$
\mathbb{V}:=\left\{f \in L_{\text {per }}^{2}([0, L]):\langle f\rangle=\frac{1}{L} \int_{0}^{L} f(x) d x=0\right\},
$$

and the orthogonal projection on $\mathbb{V}, Q: L_{\text {per }}^{2}([0, L]) \rightarrow \mathbb{V}$, given by $Q u=$ $u-\langle u\rangle$. Define $X_{m_{2}}^{0}=H_{\text {per }}^{m_{2}}([0, L]) \cap \mathbb{V}$. Then based on equation (1.8), we consider the family of closed linear operators for $\operatorname{Re} \lambda>0, \mathcal{A}^{\lambda}: X_{m_{2}}^{0} \rightarrow \mathbb{V}$ given by

$$
\mathcal{A}^{\lambda} w:=(\mathcal{M}+1) w+\frac{\partial_{x}}{\lambda-c \partial_{x}} Q\left(f^{\prime}\left(\phi_{c}\right) w-\frac{\partial_{x}}{\lambda-c \partial_{x}} w\right) .
$$

Immediately we note that $\mathcal{A}^{\lambda}$ is also well defined in $H_{\mathrm{per}}^{m_{2}}([0, L])$, and from the analyticity of the resolvent associated to the operator $\partial_{x}, \lambda \in \mathcal{S} \rightarrow(\lambda-$ $\left.c \partial_{x}\right)^{-1}$, for $\mathcal{S}=\{z \in \mathbb{C}: \operatorname{Re} z>0\}$, we obtain that $\lambda \in \mathcal{S} \rightarrow \mathcal{A}^{\lambda}$ represents an analytical family of operators of type- $A$, namely,

(1) $D\left(\mathcal{A}^{\lambda}\right)=H_{\text {per }}^{m_{2}}([0, L])$ for all $\lambda \in \mathcal{S}$, and

(2) for $u \in H_{\text {per }}^{m_{2}}([0, L]), \lambda \in \mathcal{S} \rightarrow \mathcal{A}^{\lambda} u$ is analytic in the topology of $L_{\text {per }}^{2}([0, L])$.

Therefore, we obtain that all discrete eigenvalues of $\mathcal{A}^{\lambda}(\operatorname{Re} \lambda>0)$ are stable (see Kato [15]).

In order to deduce the existence of a growing mode solution for (1.8), it is sufficient to find $\lambda \in \mathbb{C}$ with $\operatorname{Re} \lambda>0$ such that the operator $\mathcal{A}^{\lambda}$ possesses a nontrivial kernel. Indeed, for $u \in H_{\text {per }}^{m_{2}}([0, L]) \cap \mathbb{V}, u \neq 0$, such that $\mathcal{A}^{\lambda} u=0$, we obtain

$$
\begin{aligned}
0 & =\left(\lambda-c \partial_{x}\right)(\mathcal{M}+1) u+\partial_{x}\left(f^{\prime}\left(\varphi_{c}\right) u-\left\langle f^{\prime}\left(\varphi_{c}\right) u\right\rangle-\frac{\partial_{x}}{\lambda-c \partial_{x}} u\right) \\
& =\left(\lambda-c \partial_{x}\right)(\mathcal{M}+1) u+\partial_{x}\left(f^{\prime}\left(\varphi_{c}\right) u-\partial_{x}\left(\lambda-c \partial_{x}\right)^{-1} u\right) .
\end{aligned}
$$

In our approach we find a growing mode solution for $\lambda>0$ via asymptotic analytic perturbation theory. Indeed, since for

$$
\mathcal{L}_{0}=(\mathcal{M}+1)-\frac{1}{c}\left(\frac{1}{c}+f^{\prime}\left(\phi_{c}\right)\right)
$$


we have that

$$
\mathcal{A}^{\lambda} \longrightarrow Q \mathcal{L}_{0} \quad \text { as } \lambda \rightarrow 0^{+}
$$

strongly in $H_{\text {per }}^{m_{2}}([0, L]) \cap \mathbb{V}$, the usual perturbation theories do not apply, and so we extend the asymptotic perturbation arguments due to Vock and Hunziker [22] (see also Hislop and Sigal [13]) and Lin [16] to the periodic case in order to deduce the existence of a purely growing mode. In our analysis we need to count the number of eigenvalues of $\mathcal{A}^{\lambda}$ ( $\lambda$ small) in the left halfplane; since the kernel of $\mathcal{L}_{0}$ is nontrivial, we need to know how the zero eigenvalue of $Q \mathcal{L}_{0}$ is perturbed, so we deduce a moving kernel formula that allows us to decide when zero is moving to the right or left (see Lemma 4.1 below). Then, we determine the stability of all discrete eigenvalues of $Q \mathcal{L}_{0}$ when they are analytically perturbed by the operator $\mathcal{A}^{\lambda}$ for $\lambda>0$ and small enough (see Lemma 3.8).

The linearized instability result for the SRLW equation (1.3) is the following.

TheOREM 1.1 (Instability criterion for the SRLW-type equation). Define the space $X_{m_{2}}^{0}=H_{\text {per }}^{m_{2}}([0, L]) \cap \mathbb{V}$, and let $c \mapsto\left(\phi_{c}, \psi_{c}\right) \in X_{m_{2}}^{0} \times X_{m_{2}}^{0}$ be a smooth curve of periodic solutions for equation (1.5) with $c>1$. Assume that

$$
\operatorname{ker}\left(Q \mathcal{L}_{0}\right)=\left[\frac{d}{d x} \phi_{c}\right]
$$

Denote by $n^{-}\left(Q \mathcal{L}_{0}\right)$ the number (counting multiplicity) of negative eigenvalues of the operator $Q \mathcal{L}_{0}$ defined in $X_{m_{2}}^{0}$. Then there is a purely growing mode $\left(e^{\lambda t} u(x), e^{\lambda t} v(x)\right)$ with $\lambda>0, u, v \in X_{m_{2}}^{0}$, to the linearized equation (1.6) if one of the following conditions is true:

(i) $n^{-}\left(Q \mathcal{L}_{0}\right)$ is even and $I(c)<0$,

(ii) $n^{-}\left(Q \mathcal{L}_{0}\right)$ is odd and $I(c)>0$.

Here,

$$
I(c):=-\frac{1}{\left\|\phi_{c}^{\prime}\right\|_{L_{\mathrm{per}}^{2}}^{2}} \frac{1}{c} \frac{d V}{d c}
$$

with $V$ denoting the momentum evaluated in the periodic traveling wave $\phi_{c}$, that is,

$$
V(c)=\frac{1}{2}\left\langle\left(\mathcal{M}+1+\frac{1}{c^{2}}\right) \phi_{c}, \phi_{c}\right\rangle_{L_{\mathrm{per}}^{2}} .
$$


Theorem 1.1 enables us to establish a novel proof of the linear instability of cnoidal wave profiles, $c \in(1,+\infty) \rightarrow \varphi_{c}$, associated with the modified SRLW equation (henceforth, mSRLW)

$$
u_{t t}-u_{x x}+\left(3 u^{2} u_{x}\right)_{t}-u_{x x t t}=0
$$

that is, $f(u)=u^{3}$ and $\mathcal{M}=-\partial_{x}^{2}$ in equation (1.1), provided that $c \in\left(c^{*},+\infty\right)$. Moreover, our analysis shows that $c^{*}$ is a threshold value for the stability problem; namely, for $c \in\left(1, c^{*}\right)$ they are stable in $H_{\mathrm{per}}^{1}([0, L]) \times$ $L_{\text {per }}^{2}([0, L])$ (see Section 5.5 below). After that, in Section 5.6 we adapt the results due to Henry, Perez, and Wreszinski [12, Theorem 2] (see Theorem 5.13 below) to the case of dispersive equations, and we obtain that the linear instability result implies nonlinear instability. The proof that linear instability implies nonlinear instability is obtained because the mapping data-solution associated with the mSRLW equation is at least of class $C^{2}$.

Since the mSRLW equation also has a family of positive periodic solutions with a profile determined by the dnoidal Jacobi elliptic function, for the sake of completeness, we show that these periodic waves are orbitally stable in $H_{\text {per }}^{1}([0, L]) \times L_{\text {per }}^{2}([0, L])$ (see Section 5.4 below).

The following are some comments on Theorems 1.1 and 5.16 that deserve to be established. The instability criterion stated in Theorem 1.1 above is the same as the one appearing in Grillakis, Shatah, and Strauss [11, Theorem 6.2], but our conditions fall on the linear operator $Q \mathcal{L}_{0}$. Even more, our criterion does not follow directly from [11]. Indeed, in [11] it is considered the abstract Hamiltonian system

$$
\frac{d \mathbf{u}}{d t}=\mathcal{J} E^{\prime}(\mathbf{u}(t))
$$

with $\mathcal{J}$ being a one-to-one, onto, and skew-symmetric linear operator. Here, $E$ is a functional formally conserved under the flow of (1.11). In our case, the vectorial system (1.3) can be written in the form (1.11) with $\mathbf{u}=(u, v)^{t}$, $\mathcal{J}=M J_{0}$,

$$
M=\left(\begin{array}{cc}
(1+\mathcal{M})^{-1} & 0 \\
0 & I
\end{array}\right), \quad J_{0}=\left(\begin{array}{cc}
\partial_{x} & 0 \\
0 & \partial_{x}
\end{array}\right),
$$

and $E(u, v)=\int(u v-F(u)) d x$, with $F^{\prime}(u)=f(u)$. It follows immediately from the formulas for $M$ and $J_{0}$ above that $\mathcal{J}$ is neither one-to-one nor onto in our study. The linear instability criterion established in [11, Theorem 5.1] is based on the number of negative eigenvalues of a specific self-adjoint 
operator $H$ (called the linearized Hamiltonian) and the scalar function $d(c)=E\left(\phi_{c}, \psi_{c}\right)+c V\left(\phi_{c}, \psi_{c}\right)$, where $V(u, v)=(1 / 2) \int\left(u \mathcal{M} u+u^{2}+v^{2}\right) d x$. In our case, the operator $H$ is given explicitly by $H=E^{\prime \prime}\left(\phi_{c}, \psi_{c}\right)+c V^{\prime \prime}\left(\phi_{c}, \psi_{c}\right)$. In order to follow the ideas in [11] you need to determine the negative eigenvalues of $H$, which is equivalent to finding the negative eigenvalues of the operator $\mathcal{L}_{0}$ in (1.10), but our instability criterion is based on the number of negative eigenvalues of the operator $Q \mathcal{L}_{0}$. Since $\left(\phi_{c}, \psi_{c}\right)$ is a critical point for the functional $H$, it is easy to see that $d^{\prime}(c)=V\left(\phi_{c}, \psi_{c}\right)$ and consequently that

$$
d^{\prime \prime}(c)=\frac{d}{d c} V\left(\phi_{c}, \psi_{c}\right)=-c\left\|\phi_{c}^{\prime}\right\|_{L_{\mathrm{per}}^{2}}^{2} I(c) .
$$

From the last equation we have that $d^{\prime \prime}(c)$ and $I(c)$ have different signs for $c>0$, which implies by using the notation in [11] that $p\left(d^{\prime \prime}\right)=0$ if $I(c)>0$ and $p\left(d^{\prime \prime}\right)=1$ if $I(c)<0$; for this reason we can use $d^{\prime \prime}(c)$ or $I(c)$, which makes the two criteria similar. On the other hand, Theorem 5.16 below (nonlinear instability of cnoidal waves) cannot be inferred directly from the cnoidal linear instability result and from the general framework established in [11]. Indeed, the properties established above for the operator $\mathcal{J}$ are essential in [11] to deduce the nonlinear instability from a linearized instability result. In our particular case, we get around this obstacle generated by $\mathcal{J}$ by using the approach in Henry, Perez, and Wreszinski [12].

Our paper is organized as follows. In Section 2 we present notation and preliminaries. Section 3 presents the main properties of the operator $\mathcal{A}^{\lambda}$ and establishes that all the eigenvalues of $Q \mathcal{L}_{0}$ are stable by the perturbations $\mathcal{A}^{\lambda}$ for $\lambda$ small enough. Section 4 establishes the moving kernel formula and the instability proof. In Section 5, we give the theories of stability and instability of the families of traveling wave solutions with dnoidal and cnoidal profiles, respectively, for the mSRLW equation.

\section{$\S 2 . \quad$ Notation and preliminaries}

The $L^{2}$-based Sobolev spaces of periodic functions are defined as follows (for further details, see Iorio and Iorio [14]). Let $\mathcal{P}=C_{\text {per }}^{\infty}$ denote the collection of all functions $f: \mathbb{R} \rightarrow \mathbb{C}$ which are $C^{\infty}$ and periodic with period $2 L>0$. The collection $\mathcal{P}^{\prime}$ of all continuous linear functionals from $\mathcal{P}$ into $\mathbb{C}$ is the set of periodic distributions. If $\Psi \in \mathcal{P}^{\prime}$ and $\varphi \in \mathcal{P}$, we denote the value of $\Psi$ at $\varphi$ by $\langle\Psi, \varphi\rangle$. Define the functions $\Theta_{k}(x)=\exp (\pi i k x / L)$, $k \in \mathbb{Z}, x \in \mathbb{R}$. The Fourier transform of $\Psi$ is the function $\widehat{\Psi}: \mathbb{Z} \rightarrow \mathbb{C}$ defined by $\widehat{\Psi}(k)=\frac{1}{2 L}\left\langle\Psi, \Theta_{k}\right\rangle$, for all $k \in \mathbb{Z}$. So, if $\Psi$ is a periodic function with 
period $2 L$, we have $\widehat{\Psi}(k)=\frac{1}{2 L} \int_{-L}^{L} \Psi(x) e^{-\frac{i k \pi x}{L}} d x$. For $s \in \mathbb{R}$, the Sobolev space of order $s$, denoted by $H_{\text {per }}^{s}([-L, L])$, is the set of all $f \in \mathcal{P}^{\prime}$ such that $\left(1+|k|^{2}\right)^{\frac{s}{2}} \widehat{f}(k) \in l^{2}(\mathbb{Z})$, with norm $\|f\|_{H_{\text {per }}^{s}}^{2}=2 L \sum_{k=-\infty}^{\infty}\left(1+|k|^{2}\right)^{s}|\widehat{f}(k)|^{2}$. We also note that $H_{\text {per }}^{s}$ is a Hilbert space with respect to the inner product $(f \mid g)_{s}=2 L \sum_{n=-\infty}^{\infty}\left(1+|k|^{2}\right)^{s} \widehat{f}(k) \overline{\widehat{g}(k)}$. The space $H_{\text {per }}^{0}$ will be denoted by $L_{\text {per }}^{2}$ and its norm will be $\|\cdot\|_{L_{\text {per }}^{2}}$. Of course $H_{\text {per }}^{s} \subseteq L_{\text {per }}^{2}$, for any $s \geq 0$.

The normal elliptic integral of first type (see Byrd and Friedman [9]) is defined by

$$
\int_{0}^{y} \frac{d t}{\sqrt{\left(1-t^{2}\right)\left(1-k^{2} t^{2}\right)}}=\int_{0}^{\phi} \frac{d \theta}{\sqrt{1-k^{2} \sin ^{2} \theta}}=F(\phi, k),
$$

where $y=\sin \phi$ and $k \in(0,1) ; k$ is called the modulus and $\phi$ the argument. When $y=1$, we denote $F(\pi / 2, k)$ by $K=K(k)$. The Jacobian elliptic functions are denoted by $\operatorname{sn}(u ; k), \operatorname{cn}(u ; k)$, and $\operatorname{dn}(u ; k)$ (called snoidal, cnoidal, and dnoidal, respectively), and are defined via the previous elliptic integral. More precisely, let $u(y ; k):=u=F(\phi, k)$; then $y=\sin \phi:=\operatorname{sn}(u ; k)$, $\operatorname{cn}(u ; k)=\sqrt{1-\operatorname{sn}^{2}(u ; k)}$, and $\operatorname{dn}(u ; k)=\sqrt{1-k^{2} \operatorname{sn}^{2}(u ; k)}$. We have the following asymptotic formulas: $\operatorname{sn}(x ; 1)=\tanh (x), \operatorname{cn}(x ; 1)=\operatorname{sech}(x)$, and $\operatorname{dn}(x ; 1)=\operatorname{sech}(x)$.

Next, we present the Poisson summation theorem. It will be used to find the periodic traveling wave solutions for the mSRLW equation.

TheOREM 2.1. Let $\widehat{f}^{\mathbb{R}}(\xi)=\int_{-\infty}^{\infty} f(x) e^{-i x \xi} d x$ and $f(x)=$ $\int_{-\infty}^{\infty} \widehat{f}^{\mathbb{R}}(\xi) e^{i x \xi} d \xi$ satisfy

$$
|f(x)| \leq \frac{A}{(1+|x|)^{1+\delta}} \quad \text { and } \quad\left|\widehat{f}^{\mathbb{R}}(\xi)\right| \leq \frac{A}{(1+|\xi|)^{1+\delta}}
$$

where $A>0$ and $\delta>0$. Thus, for $L>0$

$$
\sum_{n=-\infty}^{\infty} f(x+2 L n)=\frac{1}{2 L} \sum_{n=-\infty}^{\infty} \widehat{f}^{\mathbb{R}}\left(\frac{n}{2 L}\right) e^{\frac{\pi i n x}{L}} .
$$

The two series above converge absolutely.

Proof. See, for example, Stein and Weiss [21, p. 250].

\section{$\S 3$. Stability of the eigenvalues of $Q \mathcal{L}_{0}$ by $\mathcal{A}^{\lambda}$}

In this section we show that all the discrete eigenvalues of $Q \mathcal{L}_{0}$ are stable by the family of linear operators $\mathcal{A}^{\lambda}$ in (1.9), for $\lambda$ positive and small enough. We begin by establishing some basic structures of the family $\mathcal{A}^{\lambda}$. 


\subsection{Properties of $\mathcal{A}^{\lambda}$}

Let us define the differential operators $\mathcal{D}=c \partial_{x}$ and $\mathcal{E}^{\lambda, \pm}=\frac{\lambda}{\lambda \pm \mathcal{D}}$. Then we can rewrite operator $\mathcal{A}^{\lambda}$ in (1.9) as

$$
\mathcal{A}^{\lambda}=(\mathcal{M}+1)-\frac{1}{c}\left(1-\mathcal{E}^{\lambda,-}\right) Q\left(f^{\prime}\left(\phi_{c}\right)+\frac{1}{c}\left(1-\mathcal{E}^{\lambda,-}\right)\right) .
$$

Proposition 3.1. For $\lambda>0$, the operator $\mathcal{A}^{\lambda}$ converges to $\mathcal{A}^{0}:=Q \mathcal{L}_{0}$ strongly in $\mathbb{V}$ when $\lambda \rightarrow 0^{+}$, and converges to $\mathcal{M}+1$ strongly in $L_{\mathrm{per}}^{2}([0, L])$ when $\lambda \rightarrow+\infty$.

Proof. Consider $\varphi \in \mathbb{V}$. Then we have

$$
c^{2}\left\|\left(\mathcal{A}^{\lambda}-Q \mathcal{L}_{0}\right) \varphi\right\|_{L_{\mathrm{per}}^{2}}^{2}=\left\|\mathcal{E}^{\lambda,-} Q\left(f^{\prime}\left(\phi_{c}\right) \varphi+\frac{2}{c} \varphi\right)+\frac{1}{c}\left(\mathcal{E}^{\lambda,-}\right)^{2} \varphi\right\|_{L_{\mathrm{per}}^{2}}^{2}
$$

and $c^{2}\left\|\left(\mathcal{A}^{\lambda}-(\mathcal{M}+1)\right) \varphi\right\|_{L_{\mathrm{per}}^{2}}^{2}=\left\|\left(\mathcal{E}^{\lambda,-}-1\right) Q\left(f^{\prime}\left(\phi_{c}\right) \varphi-\frac{1}{c}\left(\mathcal{E}^{\lambda,-}-1\right) \varphi\right)\right\|_{L_{\mathrm{per}}^{2}}^{2}$. Thus, since for $\lambda>0$ the operators $\mathcal{E}^{\lambda, \pm}$ are continuous in $L_{\text {per }}^{2}([0, L])$ with respect to $\lambda$ and satisfy the following properties (see Angulo Pava and Natali [4, Lemma 2.1]):

(i) $\left\|\mathcal{E}^{\lambda, \pm}\right\|_{B\left(L_{\text {per }}^{2}\right)} \leq 1,\left\|I-\mathcal{E}^{\lambda, \pm}\right\|_{B\left(L_{\text {per }}^{2}\right)} \leq 1$,

(ii) $\mathcal{E}^{\lambda, \pm}$ converges to 0 strongly (uniformly) in $\mathbb{V}$ as $\lambda \rightarrow 0^{+}$,

(iii) $\mathcal{E}^{\lambda, \pm}$ converges to $I$ strongly in $L_{\text {per }}^{2}([0, L])$ as $\lambda \rightarrow+\infty$,

we obtain immediately the proposition.

The next result establishes that all eigenvalues of $\mathcal{A}^{\lambda}$ (with domain $H_{\mathrm{per}}^{m_{2}}$ ) are isolated; namely, the spectrum of $\mathcal{A}^{\lambda}$ is discrete, $\sigma\left(\mathcal{A}^{\lambda}\right)=\sigma_{p}\left(\mathcal{A}^{\lambda}\right)$, and so the essential spectrum $\sigma_{\text {ess }}\left(\mathcal{A}^{\lambda}\right)$ is empty. Therefore, the spectrum of $\mathcal{A}^{\lambda}$ with domain $H_{\text {per }}^{m_{2}} \cap \mathbb{V}$ is also discrete.

Proposition 3.2. For any $\lambda>0$, we have $\sigma_{\mathrm{ess}}\left(\mathcal{A}^{\lambda}\right)=\sigma_{\mathrm{ess}}(\mathcal{M}+1)=\emptyset$.

Proof. Set $X=L_{\text {per }}^{2}([0, L]), T=\mathcal{M}+1$, and $A=-\frac{1}{c}\left(1-\mathcal{E}^{\lambda,-}\right) Q\left(f^{\prime}\left(\phi_{c}\right)+\right.$ $\left.\frac{1}{c}\left(1-\mathcal{E}^{\lambda,-}\right)\right)$. Then, $\mathcal{A}^{\lambda}=T+A$, where $D(T) \subset D(A)$, and $T$ is a closed linear operator. Therefore, since

$$
\begin{aligned}
c\|A v-A u\|_{X} & =\left\|\left(1-\mathcal{E}^{\lambda,-}\right) Q\left(f^{\prime}\left(\phi_{c}\right)+\frac{1}{c}\left(1-\mathcal{E}^{\lambda,-}\right)\right)(v-u)\right\|_{X} \\
& \leq\left(\left\|f^{\prime}\left(\phi_{c}\right)\right\|_{L_{\text {per }}^{\infty}}+\frac{1}{c}\right)\|v-u\|_{X},
\end{aligned}
$$

and $A$ is $T$-compact, Kato [15, Theorem 5.35, Chapter IV] implies that $\sigma_{\text {ess }}\left(\mathcal{A}^{\lambda}\right)=\sigma_{\text {ess }}(T)=\emptyset$. 
The next result is established for $\mathcal{A}^{\lambda}$ with domain $H_{\mathrm{per}}^{m_{2}}$.

Lemma 3.3. Let $c>1$. There exists $\Lambda>0$ such that for all $\lambda>\Lambda, \mathcal{A}^{\lambda}$ does not have eigenvalues $z \in \mathbb{C}$ satisfying $\operatorname{Re} z \leq 0$.

Proof. We follow the ideas established by Lin in [16]. Suppose by contradiction that there exists a sequence $\lambda_{n} \rightarrow+\infty$ and $\left\{b_{n}\right\}_{n \in \mathbb{N}} \subset \mathbb{C},\left\{u_{n}\right\}_{n \in \mathbb{N}} \subset$ $H_{\text {per }}^{m_{2}}$, such that $\operatorname{Re} b_{n} \leq 0$ and $\left(\mathcal{A}^{\lambda_{n}}-b_{n}\right) u_{n}=0$. Now, the inequality

$$
\begin{aligned}
\left\|\mathcal{A}^{\lambda} u-(\mathcal{M}+1) u\right\|_{L_{\mathrm{per}}^{2}}^{2} & \leq c^{-2}\left\|\left(1-\mathcal{E}^{\lambda,-}\right) Q\left(f^{\prime}\left(\phi_{c}\right) u+c^{-1}\left(1-\mathcal{E}^{\lambda,-}\right) u\right)\right\|_{L_{\mathrm{per}}^{2}} \\
& \leq C^{2}\|u\|_{L_{\mathrm{per}}^{2}}
\end{aligned}
$$

where $C>0$ does not depend on $\lambda>0$, implies for $z \in \sigma_{d}\left(\mathcal{A}^{\lambda}\right)$ and $\mathcal{A}^{\lambda} \psi=z \psi$ $(\|\psi\|=1)$ that

$$
\begin{aligned}
& (\operatorname{Re} z-\langle\psi,(\mathcal{M}+1) \psi\rangle)^{2}+(\operatorname{Im} z)^{2} \\
& \quad+\|(\mathcal{M}+1) \psi\|_{L_{\mathrm{per}}^{2}}^{2}-\langle\psi,(\mathcal{M}+1) \psi\rangle^{2} \leq C^{2}
\end{aligned}
$$

Since $\mathcal{M}+1$ is a self-adjoint positive operator, we obtain from the CauchySchwarz inequality that all eigenvalues of $\mathcal{A}^{\lambda}$ must lie in the closed subset

$$
D_{C}:=\{z \in \mathbb{C}: \operatorname{Re} z \geq-C \text { and }|\operatorname{Im} z| \leq C\} .
$$

Then, there exists $b_{\infty} \in D_{C}$ such that $b_{n} \rightarrow b_{\infty}$, as $n \rightarrow+\infty$ and $\operatorname{Re} b_{\infty} \leq 0$. Denote $e(x)=\left[f^{\prime}\left(\phi_{c}(x)\right)\right]^{2}$; thus we can normalize $u_{n}$ such that $\left\|u_{n}\right\|_{L_{\mathrm{per}}^{2}, e}:=$ $\int u_{n}^{2}(x) e(x) d x=1$. Now, since the equation $\left(\mathcal{A}^{\lambda_{n}}-b_{n}\right) u_{n}=0$ implies (see Lemma 3.7 below) $\left\|u_{n}\right\|_{H_{\text {per }}^{m_{1}}} \leq M$, where $M$ does not depend on $n$, there exists a subsequence of $\left\{u_{n}\right\}_{n \in \mathbb{N}}$, that we still denote by $\left\{u_{n}\right\}_{n \in \mathbb{N}}$, such that $u_{n} \rightarrow u_{\infty}$ in $H_{\text {per }}^{\frac{m_{1}}{2}}$, as $n \rightarrow+\infty$. On the other hand, since the embedding $H_{\text {per }}^{\frac{m_{1}}{2}} \hookrightarrow L_{\text {per }}^{2}$ is compact, we deduce that $u_{n} \longrightarrow u_{\infty}$ in $L_{\text {per }}^{2}$ and that $u_{n} \longrightarrow u_{\infty}$ in $L_{\text {per }, e}^{2}$, as $n \rightarrow+\infty$. Hence $\left\|u_{\infty}\right\|_{L_{\text {per }, e}^{2}}=1$. Next, by using Proposition 3.1 we have $\mathcal{A}^{\lambda_{n}} u_{n}-(\mathcal{M}+1) u_{n} \rightarrow 0$ as $n \rightarrow+\infty$. So, since $\mathcal{A}^{\lambda_{n}} u_{n} \rightarrow b_{\infty} u_{\infty}$, we obtain that $(\mathcal{M}+1) u_{n} \rightarrow b_{\infty} u_{\infty}$. Therefore, by $\mathcal{M}$ being a closed operator we have $u_{\infty} \in H_{\text {per }}^{m_{2}}$ and $(\mathcal{M}+1) u_{\infty}=b_{\infty} u_{\infty}$. However, since $\operatorname{Re} b_{\infty} \leq 0$, we obtain a contradiction because $\mathcal{M}+1$ is a positive operator.

Using Lemma 3.3, we obtain the following. 
Lemma 3.4. Let $c>1$. There exists $\Lambda>0$ such that for all $\lambda>\Lambda, \mathcal{A}^{\lambda}$ with domain $H_{\mathrm{per}}^{m_{2}}([0, L]) \cap \mathbb{V}$ does not have eigenvalues $z \in \mathbb{C}$ satisfying $\operatorname{Re} z \leq 0$.

\subsection{The stability of eigenvalues for $\lambda$ small enough}

In this section we study the spectra of the linear operator $\mathcal{A}^{\lambda}$ in $X_{m_{2}}^{0}$, for $0<\lambda \ll 1$, that is, $\lambda>0$ is small enough. In order to obtain the results contained in this section, we apply the arguments of asymptotic perturbation theory in Hislop and Sigal [13, Chapter 19] and Kato [15, Chapter VIII] to our periodic context. We start with the following definition. Consider the self-adjoint linear operator $Q \mathcal{L}_{0}: X_{m_{2}}^{0} \rightarrow \mathbb{V}$, given by

$$
Q \mathcal{L}_{0} g=\mathcal{L}_{0} g+\frac{1}{c L}\left\langle g, f^{\prime}\left(\phi_{c}\right)\right\rangle
$$

Definition 3.5. An eigenvalue $\mu_{0} \in \sigma\left(Q \mathcal{L}_{0}\right)=\sigma_{p}\left(Q \mathcal{L}_{0}\right)$ is stable with respect to the family $\mathcal{A}^{\lambda}$ defined in (1.9) if the following two conditions hold.

(i) There is $\delta>0$ such that the region $\mathcal{Q}_{\delta}:=\left\{z \in \mathbb{C} ; 0<\left|z-\mu_{0}\right|<\delta\right\}$ satisfies

$$
\mathcal{Q}_{\delta} \subset \rho\left(Q \mathcal{L}_{0}\right) \cap \Delta_{b},
$$

where $\rho\left(Q \mathcal{L}_{0}\right)$ is the resolvent set of $Q \mathcal{L}_{0}$, and $\Delta_{b}$ is the region of boundedness for the family $\mathcal{A}^{\lambda}$, defined by

$$
\Delta_{b}:=\left\{z \in \mathbb{C} ;\left\|R_{\lambda}(z)\right\|_{B(\mathbb{V})} \leq M, \forall 0<\lambda \ll 1\right\} .
$$

Here $M=M(z)>0$ does not depend on $\lambda$ and $R_{\lambda}(z)=\left(\mathcal{A}^{\lambda}-z\right)^{-1}$ : $\mathbb{V} \rightarrow X_{m_{2}}^{0}$.

(ii) Let $\Gamma$ be a simple closed curve about $\mu_{0}$ such that $\Gamma \subset \mathcal{Q}_{\delta} \subset \rho\left(Q \mathcal{L}_{0}\right) \cap$ $\rho\left(\mathcal{A}^{\lambda}\right)$, for all $\lambda$ small, and define the associated Riesz projector for $\mathcal{A}^{\lambda}$

$$
P_{\lambda}=-\frac{1}{2 \pi i} \int_{\Gamma} R_{\lambda}(z) d z .
$$

Then,

$$
\lim _{\lambda \rightarrow 0^{+}}\left\|P_{\lambda}-P_{\mu_{0}}\right\|_{B(\mathbb{V})}=0
$$

where $P_{\mu_{0}}$ is the Riesz projector for $Q \mathcal{L}_{0}$ and $\mu_{0}$.

REMARK 3.6. It follows from Definition 3.5 that for all $0<\lambda \ll 1$, the operators $\mathcal{A}^{\lambda}$ have discrete spectra inside the domain determined by $\Gamma$ with total algebraic multiplicity equal to that of $\mu_{0}$, because $\operatorname{dim}\left(\operatorname{Im} P_{\lambda}\right)=$ 
$\operatorname{dim}\left(\operatorname{Im} P_{\mu_{0}}\right)$ for $\lambda$ small. In order to simplify the notation, we write $\operatorname{dim}\left(P_{\lambda}\right)$ to refer to $\operatorname{dim}\left(\operatorname{Im} P_{\lambda}\right)$.

The next lemma is a periodic version of Lin [16, Lemma 2.8] and because of this, we omit its proof.

Lemma 3.7. Let $c>1$. For all $\lambda>0$ small enough, consider $u \in H_{\mathrm{per}}^{m_{2}}([0, L])$ satisfying the equation $\left(\mathcal{A}^{\lambda}-z\right) u=v$, where $z \in \mathbb{C}$ with $\operatorname{Re} z \leq \frac{1}{2}\left(1-\frac{1}{c^{2}}\right)$ and $v \in L_{\mathrm{per}}^{2}([0, L])$. Then, we have the estimative

$$
\|u\|_{H_{\mathrm{per}}^{\frac{m_{2}}{2}}} \leq M\left(\|u\|_{L_{\mathrm{per}, e}^{2}}+\|v\|_{L_{\mathrm{per}}^{2}}\right)
$$

for some constant $M>0$ which does not depend on $\lambda>0$.

The following result gives us sufficient conditions for determining when a complex number belongs to the region of boundedness for the family $\left\{\mathcal{A}^{\lambda}\right\}$.

Lemma 3.8. Let $c>1$. For $z \in \mathbb{C}$ with $\operatorname{Re} z \leq \frac{1}{2}\left(1-\frac{1}{c^{2}}\right)$, we have $z \in \Delta_{b}$ if and only if $z \in \rho\left(Q \mathcal{L}_{0}\right)$.

Proof. Let $z \in \Delta_{b}$. Then for all $u \in C_{\text {per }}^{\infty}([0, L]) \cap \mathbb{V}$, we have

$$
\left\|\left(\mathcal{A}^{\lambda}-z\right) u\right\|_{L_{\mathrm{per}}^{2}} \geq \varepsilon\|u\|_{L_{\mathrm{per}}^{2}}>0
$$

for all $0<\lambda \ll 1$, and $\varepsilon>0$ does not depend on $\lambda$. From Proposition 3.1 and (3.2) we obtain for $\lambda \rightarrow 0^{+}$that

$$
\left\|\left(Q \mathcal{L}_{0}-z\right) u\right\|_{L_{\mathrm{per}}^{2}} \geq \varepsilon\|u\|_{L_{\mathrm{per}}^{2}} .
$$

Since $Q \mathcal{L}_{0}$ is self-adjoint, it follows that $z \in \rho\left(Q \mathcal{L}_{0}\right)$.

Next, we suppose that $z \in \rho\left(Q \mathcal{L}_{0}\right)$ but $z \notin \Delta_{b}$. Then, we guarantee the existence of a sequence $\left\{u_{\lambda}\right\} \subset C_{\text {per }}^{\infty}([0, L]) \cap \mathbb{V}$, with $\left\|u_{\lambda}\right\|_{L_{\text {per }}^{2}}=1$ such that

$$
\left\|\left(\mathcal{A}^{\lambda}-z\right) u_{\lambda}\right\|_{L_{\mathrm{per}}^{2}} \longrightarrow 0, \quad \text { as } \lambda \rightarrow 0^{+} \text {. }
$$

Denote $v_{\lambda}=\left(\mathcal{A}^{\lambda}-z\right) u_{\lambda}$. So, from Lemma 3.7 we have for $\lambda$ small

$$
\left\|u_{\lambda}\right\|_{H_{\mathrm{per}}^{\frac{m_{1}}{2}}} \leq M\left(\left\|u_{\lambda}\right\|_{L_{\mathrm{per}, e}^{2}}+\left\|v_{\lambda}\right\|_{L_{\mathrm{per}}^{2}}\right) \leq K
$$

Hence, from the compact embedding $H_{\text {per }}^{m_{1} / 2} \hookrightarrow L_{\text {per }}^{2}$, we have (modulo a subsequence) that $u_{\lambda} \rightarrow u$ in $H_{\mathrm{per}}^{m_{1} / 2}$ and $u_{\lambda} \longrightarrow u$ in $\mathbb{V}$ as $\lambda \rightarrow 0^{+}$. Then 
$\|u\|_{L_{\text {per }}^{2}}=1$. Next, for any $v \in D\left(\left(\mathcal{A}^{\lambda}\right)^{*}\right)=D\left(Q \mathcal{L}_{0}\right)$, we conclude that

$$
\begin{aligned}
0 & =\lim _{\lambda \rightarrow 0^{+}}\left\langle v,\left(\mathcal{A}^{\lambda}-z\right) u_{\lambda}\right\rangle_{L_{\mathrm{per}}^{2}} \\
& =\lim _{\lambda \rightarrow 0^{+}}\left\langle\left(\left(\mathcal{A}^{\lambda}\right)^{*}-\bar{z}\right) v, u_{\lambda}\right\rangle_{L_{\mathrm{per}}^{2}}=\left\langle\left(Q \mathcal{L}_{0}-\bar{z}\right) v, u\right\rangle_{L_{\mathrm{per}}^{2}}
\end{aligned}
$$

Therefore, $u \in D\left(Q \mathcal{L}_{0}\right)$ and $\left(Q \mathcal{L}_{0}-z\right) u=0$. Since $z \in \rho\left(Q \mathcal{L}_{0}\right)$, we conclude that $u=0$. This last fact generates a contradiction because $\|u\|_{L_{\mathrm{per}}^{2}}=1$. The proof of the theorem is now completed.

Using Lemma 3.8 we obtain the following main result.

Theorem 3.9. Let $\mathcal{A}^{\lambda}$ be the linear operator defined in (1.9). Suppose that $\mu_{0} \in \sigma\left(Q \mathcal{L}_{0}\right)$ (therefore $\mu_{0}$ is a discrete eigenvalue). Then $\mu_{0}$ is stable in the sense of Definition 3.5.

Proof. Let $\mu_{0} \in \sigma\left(Q \mathcal{L}_{0}\right)$. Then we can choose $\delta>0$ such that the annular region

$$
\mathcal{Q}_{\delta}=\left\{z \in \mathbb{C}: 0<\left|z-\mu_{0}\right|<\delta\right\} \subset \rho\left(Q \mathcal{L}_{0}\right) .
$$

From Lemma 3.8, we see that $\mathcal{Q}_{\delta} \subset \Delta_{b}$. Then for $z \in \mathcal{Q}_{\delta}$

$$
\left\|R_{\lambda}(z)\right\|_{B(\mathbb{V})} \leq M, \quad \text { for } 0<\lambda \ll 1
$$

Therefore, since $\mathcal{A}^{\lambda} u \rightarrow Q \mathcal{L}_{0} u$ for $\lambda \rightarrow 0^{+}$and $\rho\left(Q \mathcal{L}_{0}\right) \cap \Delta_{b} \neq \emptyset$, from Kato [15] we have that for all $z \in \mathcal{Q}_{\delta}$ and $u \in C_{\text {per }}^{\infty}([0, L]) \cap \mathbb{V}, \lim _{\lambda \rightarrow 0^{+}} R_{\lambda}(z) u=$ $R_{0}(z) u$. Then, the strong resolvent convergence $R_{\lambda}(z) \rightarrow R_{0}(z)$ is uniform on the circle $\Gamma=\left\{z:\left|z-\mu_{0}\right|=r<\delta\right\}$. Hence the Riesz projections $P_{\lambda}$ satisfy for $u \in C_{\text {per }}^{\infty}([0, L]) \cap \mathbb{V}$ that $\lim _{\lambda \rightarrow 0^{+}} P_{\lambda} u=P_{\mu_{0}} u$, and since $P_{\mu_{0}}$ is self-adjoint, we have $\lim _{\lambda \rightarrow 0^{+}} P_{\lambda}^{*} u=P_{\mu_{0}} u$. Therefore we obtain $\operatorname{dim}\left(P_{\lambda}\right) \geq \operatorname{dim}\left(P_{\mu_{0}}\right)$ (see Kato [15, Lemma 1.23, p. 438]). Next, by using Kato [15, Lemma 1.24], the above two convergences of the Riesz projectors and the condition

$$
\operatorname{dim}\left(P_{\lambda}\right) \leq \operatorname{dim}\left(P_{\mu_{0}}\right)
$$

for all $0<\lambda \ll 1$, are sufficient to establish condition (ii) of Definition 3.5, that is, the norm convergence of the projections. Thus, let us suppose that inequality (3.4) does not occur. Then, since $P_{\mu_{0}}$ is an orthogonal projection, we can find a sequence $u_{\lambda} \in \mathbb{V},\left\|u_{\lambda}\right\|_{L_{\text {per }}^{2}}=1$ such that $P_{\lambda} u_{\lambda}=u_{\lambda}$ and $P_{\mu_{0}} u_{\lambda}=0$. Hence, there is a subsequence, still denoted by $\left\{u_{\lambda}\right\}$, such 
that $u_{\lambda} \rightarrow u_{0}$ in $L_{\text {per }}^{2}$. Now, for $v \in L_{\text {per }}^{2}$ and $\lambda \rightarrow 0^{+}$the relation $\left\langle v, u_{\lambda}\right\rangle=$ $\left\langle v,\left(P_{\lambda}-P_{\mu_{0}}\right) u_{\lambda}\right\rangle=\left\langle\left(P_{\lambda}^{*}-P_{\mu_{0}}\right) v, u_{\lambda}\right\rangle$ implies that $\left\langle v, u_{0}\right\rangle=0$ and so, $u_{0}=0$.

On the other hand, for $z \in \mathcal{Q}_{\delta}-\Gamma$ we have from the first resolvent identity that

$$
\left(\mathcal{A}^{\lambda}-z\right) P_{\lambda} u_{\lambda}=-\frac{1}{2 \pi i} \int_{\Gamma}\left[u_{\lambda}-(z-\eta) R_{\lambda}(\eta) u_{\lambda}\right] d \eta
$$

Therefore, from (3.3) and the compactness of $\Gamma$, we obtain for $0<\lambda \ll 1$ that

$$
\left\|\left(\mathcal{A}^{\lambda}-z\right) P_{\lambda} u_{\lambda}\right\|_{L_{\mathrm{per}}^{2}} \leq M_{0}\left[1+\sup _{\eta \in \Gamma}|z-\eta|\right] .
$$

Hence,

$$
\left\|\mathcal{A}^{\lambda} u_{\lambda}\right\|_{L_{\mathrm{per}}^{2}} \leq\left\|\left(\mathcal{A}^{\lambda}-z\right) P_{\lambda} u_{\lambda}\right\|_{L_{\mathrm{per}}^{2}}+\left\|z P_{\lambda} u_{\lambda}\right\|_{L_{\mathrm{per}}^{2}} \leq M
$$

where $M>0$ does not depend on $\lambda>0$. Inequality (3.5) implies that $u_{\lambda}$ is bounded in $H_{\text {per }}^{m_{2}}$. So, we obtain (modulo a subsequence) that there is $u \in L_{\text {per }}^{2}$ such that $u_{\lambda} \longrightarrow u$ in $L_{\text {per }}^{2}$, as $\lambda \rightarrow 0^{+}$, with $\|u\|_{L_{\text {per }}^{2}}=1$. Since $u_{\lambda}$ converges weakly to zero in $L_{\text {per }}^{2}$, we obtain a contradiction from the uniqueness of the weak limit. This finishes the proof of the theorem.

\section{$\S 4$. The moving kernel formula and the instability proof}

In this section we study the perturbation of the eigenvalue $\mu=0$ associated with the linear operator $Q \mathcal{L}_{0}$ with respect to the operator $\mathcal{A}^{\lambda}$ for small $\lambda>0$. For this purpose we derive a moving kernel formula in order to determine an instability criterion. Let us suppose that $\operatorname{ker}\left(Q \mathcal{L}_{0}\right)=\operatorname{ker}\left(\mathcal{L}_{0}\right)=$ $\left[\frac{d}{d x} \varphi_{c}\right]$. Then, $\operatorname{dim} P_{0}=1$ and from Theorem 3.9 one has $\operatorname{dim} P_{\lambda}=1$ for all $0<\lambda \ll 1$. We note that since the eigenvalues of $\mathcal{A}^{\lambda}$ appear in conjugate pairs, we have that there is only one real eigenvalue $b_{\lambda}$ of $\mathcal{A}^{\lambda}$ inside $B(0 ; \delta)$. The idea in the next result is to determine the sign of $b_{\lambda}$, for $\lambda$ small.

LEMMA 4.1. Let $c>1$, and assume that $\operatorname{ker}\left(Q \mathcal{L}_{0}\right)=\left[\frac{d}{d x} \phi_{c}\right]$. For $\lambda>0$ small enough, let $b_{\lambda} \in \mathbb{R}$ be the only eigenvalue of $\mathcal{A}^{\lambda}$ near origin. Then,

$$
\lim _{\lambda \rightarrow 0^{+}} \frac{b_{\lambda}}{\lambda^{2}}=I(c):=-\frac{1}{\left\|\phi_{c}^{\prime}\right\|_{L_{\mathrm{per}}^{2}}^{2}} \frac{1}{c} \frac{d V}{d c}
$$

with $V$ denoting the momentum evaluated in the periodic traveling wave $\phi_{c}$, that is,

$$
V(c)=\frac{1}{2}\left\langle\left(\mathcal{M}+1+\frac{1}{c^{2}}\right) \phi_{c}, \phi_{c}\right\rangle_{L_{\mathrm{per}}^{2}} .
$$


Proof. From Theorem 3.9 we see that for $\lambda>0$ small enough, there exists $u_{\lambda} \in H_{\text {per }}^{m_{2}}([0, L]) \cap \mathbb{V}$ such that $\left(\mathcal{A}^{\lambda}-b_{\lambda}\right) u_{\lambda}=0, b_{\lambda} \in \mathbb{R}$, and $\lim _{\lambda \rightarrow 0^{+}} b_{\lambda}=0$. We set $\left\|u_{\lambda}\right\|_{L_{\text {per }}^{2}, e}=1$. So, from Lemma 3.7 we have that $\left\|u_{\lambda}\right\|_{H_{\mathrm{per}}}^{\frac{m_{2}}{2}} \leq C$, for some constant $C>0$ which does not depend on $\lambda>0$. Then, modulo a subsequence, we have that $u_{\lambda} \rightarrow u_{0}$ in $H_{\text {per }}^{\frac{m_{2}}{2}}([0, L])$, as $\lambda \rightarrow 0^{+}$, and that

$$
u_{\lambda} \longrightarrow u_{0} \quad \text { in } \mathbb{V} \text {, as } \lambda \rightarrow 0^{+} \text {. }
$$

Then, since $\mathcal{A}^{0} u_{0}=Q \mathcal{L}_{0} u_{0}$ and $\operatorname{ker}\left(Q \mathcal{L}_{0}\right)=\left[\phi_{c}^{\prime}\right]$, we guarantee the existence of $c_{0} \neq 0$ such that $u_{0}=c_{0} \phi_{c}^{\prime}$. We can assume that $c_{0}=1$ by normalizing the sequence. Moreover, from the equality $\left(\mathcal{A}^{\lambda}-b_{\lambda}\right)\left(u_{\lambda}-u_{0}\right)=b_{\lambda} u_{0}+\left(\mathcal{A}^{0}-\right.$ $\left.\mathcal{A}^{\lambda}\right) u_{0}$, we obtain from Lemma 3.7, Proposition 3.1, and (4.2) that $u_{\lambda} \rightarrow u_{0}$ in $H_{\text {per }}^{\frac{m_{2}}{2}}([0, L])$ as $\lambda \rightarrow 0^{+}$.

Next, we show that $\lim _{\lambda \rightarrow 0^{+}} \frac{b_{\lambda}}{\lambda}=0$. Indeed, since $\left(\mathcal{A}^{\lambda}-b_{\lambda}\right) u_{\lambda}=0$, we obtain

$$
\frac{b_{\lambda}}{\lambda} u_{\lambda}=\frac{1}{\lambda} \mathcal{A}^{\lambda} u_{\lambda}=\frac{1}{\lambda} \mathcal{A}^{0} u_{\lambda}+\frac{\mathcal{A}^{\lambda}-\mathcal{A}^{0}}{\lambda} u_{\lambda} .
$$

Then, $\mathcal{A}^{0} \phi_{c}^{\prime}=0$ implies that $\frac{b_{\lambda}}{\lambda}\left\langle u_{\lambda}, \phi_{c}^{\prime}\right\rangle=\left\langle\frac{\mathcal{A}^{\lambda}-\mathcal{A}^{0}}{\lambda} u_{\lambda}, \phi_{c}^{\prime}\right\rangle$. Using the argument in Proposition 3.1 and from the formula

$$
\begin{aligned}
\left\langle\frac{\mathcal{A}^{\lambda}-\mathcal{A}^{0}}{\lambda} u_{\lambda}, \phi_{c}^{\prime}\right\rangle= & \frac{1}{c^{2}}\left\langle\left(1-\mathcal{E}^{\lambda,-}\right) Q\left[\frac{1}{c}+f^{\prime}\left(\phi_{c}\right)\right] u_{\lambda}, \phi_{c}\right\rangle \\
& +\frac{1}{c^{3}}\left\langle\left(1-\mathcal{E}^{\lambda,-}\right)^{2} u_{\lambda}, \phi_{c}\right\rangle
\end{aligned}
$$

we conclude that

$$
\lim _{\lambda \rightarrow 0^{+}} \frac{b_{\lambda}}{\lambda}=\frac{1}{c^{2}\left\|\phi_{c}^{\prime}\right\|^{2}}\left\langle Q\left[\frac{1}{c}+f^{\prime}\left(\phi_{c}\right)\right] \phi_{c}^{\prime}, \phi_{c}\right\rangle+\frac{1}{c^{3}}\left\langle\phi_{c}^{\prime}, \phi_{c}\right\rangle=0 .
$$

Next, we calculate $\lim _{\lambda \rightarrow 0^{+}} \frac{b_{\lambda}}{\lambda^{2}}$. We write $u_{\lambda}=c_{\lambda} \phi_{c}^{\prime}+\lambda v_{\lambda}$, with $c_{\lambda}=\frac{\left\langle u_{\lambda}, \phi_{c}^{\prime}\right\rangle}{\left\|\phi_{c}^{\prime}\right\|^{2}}$. Then $\left\langle v_{\lambda}, \phi_{c}^{\prime}\right\rangle=0$ and $c_{\lambda} \rightarrow 1$ as $\lambda \rightarrow 0^{+}$. Next, we follow the same strategy as in Lin [16] to obtain the bound

$$
\left\|v_{\lambda}\right\|_{H_{\mathrm{per}}^{\frac{m_{2}}{2}}} \leq C
$$

where $C>0$ does not depend on $\lambda>0$. Indeed, first note that

$$
\mathcal{A}^{\lambda} v_{\lambda}=\frac{b_{\lambda}}{\lambda} u_{\lambda}-c_{\lambda} \frac{\mathcal{A}^{\lambda} \phi_{c}^{\prime}}{\lambda}=\frac{b_{\lambda}}{\lambda} u_{\lambda}-c_{\lambda}\left(\frac{\mathcal{A}^{\lambda}-\mathcal{A}^{0}}{\lambda}\right) \phi_{c}^{\prime} .
$$


So, by denoting $w_{\lambda}:=\left(\frac{\mathcal{A}^{\lambda}-\mathcal{A}^{0}}{\lambda}\right)$, we get that

$$
w_{\lambda} \phi_{c}^{\prime}=\frac{1}{c^{2}}\left(\mathcal{E}^{\lambda,-}-1\right)\left[\frac{1}{c} \phi_{c}+f\left(\phi_{c}\right)\right]-\frac{1}{c^{3}}\left(\mathcal{E}^{\lambda,-}-1\right)^{2} \phi_{c}
$$

and we can prove that $\left\|w_{\lambda}\right\|_{L_{\mathrm{per}}^{2}} \leq C$, for some $C>0$ which does not depend on $\lambda>0$. Since $\left\langle\phi_{c}\right\rangle=0$ and

$$
(\mathcal{M}+1) \phi_{c}-\frac{1}{c}\left[\frac{1}{c} \phi_{c}+f\left(\phi_{c}\right)\right]=A_{\phi_{c}}=0,
$$

we obtain that $\left\langle\frac{1}{c} \phi_{c}+f\left(\phi_{c}\right)\right\rangle=0$, and consequently

$$
\omega_{\lambda} \phi_{c}^{\prime} \longrightarrow-\frac{1}{c}(\mathcal{M}+1) \phi_{c}-\frac{1}{c^{3}} \phi_{c}, \quad \text { as } \lambda \rightarrow 0^{+} .
$$

Therefore, if we combine (4.4), (4.8), Proposition 3.1, and Lemma 3.7, we get the desired result in (4.5). Thus $v_{\lambda} \rightarrow v_{0}$ in $H_{\mathrm{per}}^{\frac{m_{2}}{2}}([0, L])$ and $v_{\lambda} \rightarrow v_{0}$ in $\mathbb{V}$, as $\lambda \rightarrow 0^{+}$. From (4.6) and (4.8) we obtain the equality $Q \mathcal{L}_{0} v_{0}=$ $\frac{1}{c}(\mathcal{M}+1) \phi_{c}+\frac{1}{c^{3}} \phi_{c}$. Now, from (4.7) we have

$$
\mathcal{L}_{0}\left(\frac{d}{d c} \phi_{c}\right)=-\frac{1}{c}(\mathcal{M}+1) \phi_{c}-\frac{1}{c^{3}} \phi_{c}
$$

that is, $Q \mathcal{L}_{0}\left(v_{0}+\frac{d}{d c} \phi_{c}\right)=0$. From $\operatorname{ker}\left(Q \mathcal{L}_{0}\right)=\left[\phi_{c}^{\prime}\right]$, there is $\theta \in \mathbb{R}$ such that $v_{0}+\frac{d}{d c} \phi_{c}=\theta \phi_{c}^{\prime}$. Next, defining $\overline{c_{\lambda}}:=c_{\lambda}+\lambda \theta$ and $\overline{v_{\lambda}}:=v_{\lambda}-\theta \phi_{c}^{\prime}$ we obtain

$$
u_{\lambda}=\overline{c_{\lambda}} \phi_{c}^{\prime}+\lambda \overline{v_{\lambda}} .
$$

From the limit $v_{\lambda} \rightarrow v_{0}$ in $L_{\text {per }, e}^{2}([0, L])$ and the fact that

$$
\left(\mathcal{A}^{\lambda}-b_{\lambda}\right)\left(v_{\lambda}-v_{0}\right)=\frac{b_{\lambda}}{\lambda} u_{\lambda}-c_{\lambda} \omega_{\lambda}-\mathcal{A}^{\lambda} v_{0}-b_{\lambda} v_{\lambda}+b_{\lambda} v_{0} \longrightarrow 0,
$$

in $L_{\mathrm{per}}^{2}([0, L])$, as $\lambda \rightarrow 0^{+}$, we obtain from Lemma 3.7 that $\left\|v_{\lambda}-v_{0}\right\|_{H_{\mathrm{per}}} \frac{m_{2}}{2} \rightarrow$ 0 , as $\lambda \rightarrow 0^{+}$. Then, $\overline{v_{\lambda}} \rightarrow v_{0}-\theta \phi_{c}^{\prime}=-\frac{d}{d c} \phi_{c}$, as $\lambda \rightarrow 0^{+}$. Moreover, from (4.3) and (4.9)

$$
J(\lambda):=\left\langle\frac{b_{\lambda}}{\lambda^{2}} u_{\lambda}, \phi_{c}^{\prime}\right\rangle=\frac{1}{\lambda^{2}}\left\langle Q \mathcal{L}_{0} u_{\lambda}, \phi_{c}^{\prime}\right\rangle+\frac{\overline{c_{\lambda}}}{\lambda}\left\langle\omega_{\lambda} \phi_{c}^{\prime}, \phi_{c}^{\prime}\right\rangle+\left\langle\omega_{\lambda} \overline{v_{\lambda}}, \phi_{c}^{\prime}\right\rangle .
$$

Next, we estimate the last two terms in (4.10) for $\lambda \rightarrow 0^{+}$because the first 
one is zero. By using the argument in Proposition 3.1, we obtain

$$
\begin{aligned}
\left\langle w_{\lambda} \overline{v_{\lambda}}, \phi_{c}^{\prime}\right\rangle & =\frac{1}{c^{2}}\left\langle\left(1-\mathcal{E}^{\lambda,-}\right) Q\left[\frac{1}{c}+f^{\prime}\left(\phi_{c}\right)\right] \overline{v_{\lambda}}, \phi_{c}\right\rangle+\frac{1}{c^{3}}\left\langle\left(1-\mathcal{E}^{\lambda,-}\right)^{2} \overline{v_{\lambda}}, \phi_{c}\right\rangle \\
& \longrightarrow-\frac{1}{c^{2}}\left\langle\left[\frac{1}{c}+f^{\prime}\left(\phi_{c}\right)\right] \frac{d}{d c} \phi_{c}, \phi_{c}\right\rangle-\frac{1}{c^{3}}\left\langle\frac{d}{d c} \phi_{c}, \phi_{c}\right\rangle
\end{aligned}
$$

and

$$
\begin{aligned}
\left\langle\frac{w_{\lambda}}{\lambda} \phi_{c}^{\prime}, \phi_{c}^{\prime}\right\rangle= & \frac{1}{c}\left\langle\frac{c \partial_{x}}{\lambda\left(\lambda-c \partial_{x}\right)}(\mathcal{M}+1) \phi_{c}, \phi_{c}^{\prime}\right\rangle-\frac{1}{\lambda c^{3}}\left\langle\left(\frac{c \partial_{x}}{\lambda-c \partial_{x}}\right)^{2} \phi_{c}, \phi_{c}^{\prime}\right\rangle \\
= & \frac{1}{c^{2}}\left\langle\left(1-\mathcal{E}^{\lambda,-}\right)(\mathcal{M}+1) \phi_{c}, \phi_{c}\right\rangle+\frac{1}{c^{4}}\left\langle\left(1-\mathcal{E}^{\lambda,-}\right) \phi_{c}, \phi_{c}\right\rangle \\
& +\frac{1}{c^{4}}\left\langle\left(1-\mathcal{E}^{\lambda,-}\right)^{2} \phi_{c}, \phi_{c}\right\rangle \\
\longrightarrow & \frac{1}{c^{2}}\left\langle(\mathcal{M}+1) \phi_{c}, \phi_{c}\right\rangle+\frac{2}{c^{4}}\left\langle\phi_{c}, \phi_{c}\right\rangle .
\end{aligned}
$$

Then, from the equality

$$
(\mathcal{M}+1) \frac{d}{d c} \phi_{c}=-\frac{1}{c}(\mathcal{M}+1) \phi_{c}+\frac{1}{c}\left[\frac{1}{c}+f^{\prime}\left(\phi_{c}\right)\right] \frac{d}{d c} \phi_{c}-\frac{1}{c^{3}} \phi_{c},
$$

we obtain from (4.10)

$$
\begin{aligned}
\lim _{\lambda \rightarrow 0^{+}} J(\lambda) & =\frac{1}{c}\left\langle-(\mathcal{M}+1) \frac{d}{d c} \phi_{c}-\frac{1}{c^{2}} \frac{d}{d c} \phi_{c}+\frac{1}{c^{3}} \phi_{c}, \phi_{c}\right\rangle \\
& =-\frac{1}{c}\left\langle\left(\mathcal{M}+1+\frac{1}{c^{2}}\right) \frac{d}{d c} \phi_{c}, \phi_{c}\right\rangle+\frac{1}{c^{4}}\left\langle\phi_{c}, \phi_{c}\right\rangle .
\end{aligned}
$$

Thus from (4.1)

$$
\lim _{\lambda \rightarrow 0^{+}} \frac{b_{\lambda}}{\lambda^{2}}=\lim _{\lambda \rightarrow 0^{+}} \frac{J(\lambda)}{\left\langle u_{\lambda}, \phi_{c}^{\prime}\right\rangle}=I(c) .
$$

The next lemma gives a sufficient condition to obtain the relation $\operatorname{ker}\left(Q \mathcal{L}_{0}\right)=\operatorname{ker}\left(\mathcal{L}_{0}\right)$. The proof is immediate.

Lemma 4.2. Consider the operator $\mathcal{L}_{0}: H_{\mathrm{per}}^{m_{2}}([0, L]) \rightarrow L_{\mathrm{per}}^{2}([0, L])$ defined in (1.10). If for all $u \in \mathcal{L}_{0}^{-1}(1)$ we have that $\langle u\rangle=0$, then the operator $Q \mathcal{L}_{0}: X_{m_{2}}^{0} \rightarrow \mathbb{V}$ satisfies $\operatorname{ker}\left(Q \mathcal{L}_{0}\right)=\operatorname{ker}\left(\mathcal{L}_{0}\right)$.

The proof of the next theorem is obtained following the ideas of Lin [16] and Angulo Pava and Natali [4]; we make it here just for the sake of completeness. 
Proof of Theorem 1.1 (Instability criterion for the SRLW-type equation). We sketch the proof assuming (ii) since the arguments can be mimicked if we suppose (i). Assume that $n^{-}\left(Q \mathcal{L}_{0}\right)$ is odd and that $I(c)>0$. Consider $k_{1}^{-}, k_{2}^{-}, \ldots, k_{l}^{-}$, with $l \leq n^{-}\left(Q \mathcal{L}_{0}\right)$, all the distinct negative eigenvalues of $Q \mathcal{L}_{0}$. Since eigenvalues $k_{i}^{-}, i=1,2, \ldots, l$ are isolated, we guarantee the existence of $\delta_{1}>0$ such that the $l$ open disks $B_{\delta_{1}}\left(k_{i}^{-}\right)$are disjoint still lie in the left half-plane $(\operatorname{Re} z<0)$. Using Theorem 3.9, there exists $\lambda_{1}>0$ and $\delta>0$ small enough, $\delta<\delta_{1}$, such that for $0<\lambda<\lambda_{1}, \mathcal{A}^{\lambda}$ has $n^{-}\left(Q \mathcal{L}_{0}\right)$ eigenvalues (counting multiplicity) in $\bigcup_{i=1}^{l} B_{\delta}\left(k_{i}^{-}\right)$. Since $I(c)>0$, we see from Lemma 4.1 that the zero eigenvalue of $\mathcal{A}^{0}=Q \mathcal{L}_{0}$ is perturbed to a positive eigenvalue $0<b_{\lambda}<\delta$ of $\mathcal{A}^{\lambda}$ for small $\lambda_{0}$. Let us consider the region $D_{C}^{0}:=\{z \in \mathbb{C}:-2 C<\operatorname{Re} z<0$ and $|\operatorname{Im} z|<2 C\}$, where $C>0$ is the same constant which appears in Lemma 3.3. Then if we repeat the same arguments as in Lemma 3.3, we get that $\mathcal{A}^{\lambda}$ has exactly $n^{-}\left(Q \mathcal{L}_{0}\right)+1$ eigenvalues (counting multiplicity) in $D_{C}^{0}:=\{z \in \mathbb{C}:-2 C<\operatorname{Re} z<2 \delta$ and $|\operatorname{Im} z|<2 C\}$. Then, all eigenvalues of $\mathcal{A}^{\lambda}$ with real part no greater than $2 \delta$ lie in $\bigcup_{i=1}^{l} B_{\delta}\left(k_{i}^{-}\right) \cup B_{\delta}(0)$. Thus for small $\lambda, \mathcal{A}^{\lambda}$ has exactly $n^{-}\left(Q \mathcal{L}_{0}\right)$ eigenvalues in $D_{C}^{0}$.

Now, we assume that the conclusion of the theorem does not occur. Then, $\operatorname{Ker}\left(\mathcal{A}^{\lambda}\right)=\{0\}$ for any $\lambda>0$. Let $n_{D_{C}^{0}}(\lambda)$ be the number of eigenvalues (with multiplicity) of $\mathcal{A}^{\lambda}$ in $D_{C}^{0}$. Since the spectrum of $\mathcal{A}^{\lambda}$ is discrete and $\overline{D_{C}^{0}}$ is compact, we conclude that $n_{D_{C}^{0}}(\lambda)$ is always a finite integer for every $\lambda>0$. Moreover, for $\lambda>0$ small enough $n_{D_{C}^{0}}(\lambda)=n^{-}\left(Q \mathcal{L}_{0}\right)$ is odd and there is $\Lambda>0$ such that for $\lambda>\Lambda$, we conclude that $n_{D_{C}^{0}}(\lambda)=0$. Next, we define the two nonempty sets $S_{\text {odd }}=\left\{\lambda>0 ; n_{D_{C}^{0}}(\lambda)\right.$ is odd $\}$ and $S_{\text {even }}=$ $\left\{\lambda>0 ; n_{D_{C}^{0}}(\lambda)\right.$ is even $\}$. Then since the complex eigenvalues of $\mathcal{A}^{\lambda}$ appear in conjugate pairs, the number of pure complex eigenvalues is even (since $\left.\operatorname{Ker}\left(\mathcal{A}^{\lambda}\right)=\{0\}\right)$, and since the mapping $\lambda \in(0,+\infty) \rightarrow \mathcal{A}^{\lambda}$ is analytic, we can conclude that both $S_{\text {odd }}$ and $S_{\text {even }}$ are open disjoint subsets such that $(0,+\infty)=S_{\text {odd }} \cup S_{\text {even }}$, which is a contradiction.

So, there exists $\lambda>0$ and $0 \neq u \in X_{m_{2}}^{0}$ such that $\mathcal{A}^{\lambda} u=0$, and therefore $e^{\lambda t} u(x)$ is a purely growing mode solution to (1.8). With this solution in hand, it is easy to obtain a solution for (1.7) of the form $\left(e^{\lambda t} u(x), e^{\lambda t} v(x)\right)$. This finishes the proof of the theorem. 


\section{$\S 5$. Stability and instability for the MSRLW equation}

In this section we study the stability and instability of two families of periodic traveling wave solutions for the mSRLW equation, namely,

$$
u_{t t}-u_{x x}+\left(3 u^{2} u_{x}\right)_{t}-u_{x x t t}=0
$$

Our analysis of the stability makes use of the classical ideas in Benjamin [6], Bona [7], and Weinstein [23]. For linear instability we apply the results established in the previous sections.

The mSRLW equation is equivalent to

$$
\left\{\begin{array}{l}
u_{t}-u_{x x t}+3 u^{2} u_{x}-v_{x}=0 \\
v_{t}-u_{x}=0
\end{array}\right.
$$

So, suppose that $(u, v)$, with $u(x, t)=\phi_{c}(x-c t)$ and $v(x, t)=\psi(x-c t)$, is a solution for $(5.1)$. Then $\left(\phi_{c}, \psi_{c}\right)$ satisfy

$$
\left\{\begin{array}{l}
c \phi_{c}^{\prime \prime}-c \phi_{c}+\phi_{c}^{3}-\psi_{c}=0 \\
-c \psi_{c}-\phi_{c}=0
\end{array}\right.
$$

where we considered all the constants of integration equal to zero. Using the last system, we have that the profile $\phi_{c}$ satisfies

$$
c^{2} \phi_{c}^{\prime \prime}-\left(c^{2}-1\right) \phi_{c}+c \phi_{c}^{3}=0
$$

Two Jacobian elliptic profile solutions of (5.3) are of important interest in applications, the dnoidal and cnoidal solutions. For the dnoidal profile we use the Poisson summation theorem, and for the cnoidal profile we use the classical quadrature method.

\subsection{Dnoidal solutions}

Here we apply a similar argument as in Angulo Pava, Banquet, and Scialom [3, Section 3] to establish the existence of a smooth curve of positive, even, and periodic traveling wave solutions for the mSRLW equation. So, we start by considering the solitary wave solutions for equation (5.3)

$$
\varphi_{w}(x)=\sqrt{\frac{2\left(w^{2}-1\right)}{w}} \operatorname{sech}\left(\sqrt{\frac{w^{2}-1}{w^{2}} x}\right), \quad w>1, x \in \mathbb{R},
$$


with Fourier transform $\widehat{\varphi}_{w}^{\mathbb{R}}(\xi)=\sqrt{2 w} \pi \operatorname{sech}\left(\frac{\pi \xi}{2} \sqrt{\frac{w^{2}}{w^{2}-1}}\right)$. Then, from Theorem 2.1 we obtain the following periodic function

$$
\theta_{w}(x):=\frac{\sqrt{2 w} \pi}{L}+\frac{2 \sqrt{2 w} \pi}{L} \sum_{n=1}^{\infty} \operatorname{sech}\left(\frac{\pi n}{2 L} \sqrt{\frac{w^{2}}{w^{2}-1}}\right) \cos \left(\frac{2 \pi n x}{L}\right)
$$

where $w>1$ will be chosen later for $\theta_{w}$ to be a periodic traveling wave of (5.3). On the other hand, we have the Fourier expansion of the dnoidal Jacobi elliptic function of period $L$ (see Oberhettinger [18])

$$
\frac{2 K}{L} \operatorname{dn}\left(\frac{2 K \xi}{L} ; k\right)=\frac{\pi}{L}+\frac{2 \pi}{L} \sum_{n=1}^{\infty} \operatorname{sech}\left(\frac{n \pi K^{\prime}}{K}\right) \cos \left(\frac{2 n \pi \xi}{L}\right),
$$

where $K=K(k)$ is the complete elliptic integral of the first kind and $K^{\prime}(k) \equiv K\left(\sqrt{1-k^{2}}\right)$. Now, because of the shape of the series that determines $\theta_{w}$, we consider the profile

$$
\phi_{c}(x)=\eta \operatorname{dn}\left(\frac{\eta x}{\sqrt{2 c}} ; k\right)
$$

with $\eta>0$ and $k \in(0,1)$ fixed, a periodic solution (with period $L$ ) for the equation (5.3). Then, substituting this type of solution in (5.3) and using the fact that the fundamental period of the dnoidal function is $2 K$, we obtain the following compatibility relations

$$
\frac{c^{2}-1}{c^{2}}=\frac{\eta^{2}}{2}\left(2-k^{2}\right) \quad \text { and } \quad \eta=\frac{2 \sqrt{2 c} K(k)}{L} .
$$

Thus, for $k \in(0,1)$ we should have that $\eta \in\left(\sqrt{\frac{c^{2}-1}{c}}, \sqrt{\frac{2\left(c^{2}-1\right)}{c}}\right)$ and $\frac{c^{2}-1}{c^{2}}>\frac{2 \pi^{2}}{L^{2}}$ (the last one because $K(k)>\pi / 2$ for all $k$ ). Combining the two equations given in (5.6) it is easy to see that

$$
L^{2}=\frac{4 c^{2}\left(2-k^{2}\right) K^{2}(k)}{c^{2}-1},
$$

and since $c>1$, we obtain the a priori estimate $L>\sqrt{2} \pi$. The compatibility relations in (5.6) also imply that

$$
c^{2}=\frac{L^{2}}{L^{2}-4\left(2-k^{2}\right) K^{2}(k)} .
$$


Using again the assumption $c>1$, we should have that there exists $k_{L} \in(0,1)$ such that

$$
L^{2}-4\left(2-k^{2}\right) K^{2}(k)>0, \quad \text { for all } k \in\left(0, k_{L}\right) .
$$

Next, if we consider $\phi_{c}$ with fundamental period $T_{\phi_{c}}$, we obtain from (5.6) that it can be seen as a function of $\eta$ given by

$$
T_{\phi_{c}}(\eta)=2 K(k) \sqrt{2-k^{2}} \sqrt{\frac{c^{2}}{c^{2}-1}},
$$

with $k^{2}=2-\frac{2\left(c^{2}-1\right)}{\eta^{2} c}$. So, if $\eta \rightarrow \sqrt{\frac{c^{2}-1}{c}}$, then $k \rightarrow 0^{+} ;$therefore $T_{\phi_{c}}(\eta) \rightarrow$ $\sqrt{2} \pi \sqrt{\frac{c^{2}}{c^{2}-1}}$. If $\eta \rightarrow \sqrt{\frac{2\left(c^{2}-1\right)}{c}}$, then $k \rightarrow 1^{-} ;$therefore $T_{\phi_{c}}(\eta) \rightarrow+\infty$. Since $\eta \mapsto T_{\phi_{c}}(\eta)$ is a strictly increasing function (we prove it later), we obtain that $T_{\phi_{c}}(\eta)>\sqrt{2} \pi \sqrt{\frac{c^{2}}{c^{2}-1}}$.

Since the proof of the next theorem and corollaries follows the same lines as in Angulo Pava, Banquet, and Scialom [3], we omit the details.

TheOREM 5.1. Let $L>\sqrt{2} \pi$ be fixed, and let $k_{L} \in(0,1)$ satisfying (5.7). Consider $c_{0}>\frac{L}{\sqrt{L^{2}-2 \pi^{2}}}$ and the unique $\eta_{0}=\eta\left(c_{0}\right)$ such that $T_{\phi_{c_{0}}}\left(\eta_{0}\right)=L$. Then we have the following.

(i) There exist an interval $I\left(c_{0}\right)$ around $c_{0}$, an interval $J\left(\eta_{0}\right)$ around $\eta_{0}$, and a unique smooth function $\Lambda: I\left(c_{0}\right) \longrightarrow J\left(\eta_{0}\right)$ such that $\Lambda\left(c_{0}\right)=\eta_{0}$ and

$$
2 \sqrt{2-k^{2}} K(k) \sqrt{\frac{c^{2}}{c^{2}-1}}=L,
$$

where $c \in I\left(c_{0}\right), \eta=\Lambda(c) \in J\left(\eta_{0}\right)$, and $k=k(c)$ is given by $k^{2}=2-$ $\frac{2\left(c^{2}-1\right)}{\eta^{2} c}$.

(ii) The wave solution $\left(\phi_{c}, \psi_{c}\right)$, where $-c \psi_{c}=\phi_{c}$ and $\phi_{c}$ is given by (5.5), determined by $\eta=\eta(c)$, has fundamental period $L$ and satisfies (5.2). Furthermore, the mapping

$$
c \in I\left(c_{0}\right) \longmapsto\left(\phi_{c}, \psi_{c}\right) \in H_{\mathrm{per}}^{n}([0, L]) \times H_{\mathrm{per}}^{n}([0, L])
$$

is smooth for all $n \in \mathbb{N}$.

(iii) $I\left(c_{0}\right)$ can be chosen as $\mathcal{I}=\left(\frac{L}{\sqrt{L^{2}-2 \pi^{2}}},+\infty\right)$.

In the next corollary we choose the speed $w=w(c)$ in such way that $\theta_{w}$ in (5.4) becomes a periodic traveling wave with dnoidal profile. 
Corollary 5.2. Define

$$
w^{2}(c)=\frac{16 c^{2}\left(2-k^{2}\right) K^{\prime 2}}{16 c^{2}\left(2-k^{2}\right) K^{\prime 2}-c^{2}+1},
$$

where $k=k(c) \in\left(0, k_{L}\right)$ and $c>\frac{L}{\sqrt{L^{2}-2 \pi^{2}}}$. Then $\phi_{c}=\sqrt{\frac{c}{w(c)}} \psi_{w(c)}$.

Corollary 5.3. Consider the mapping $\Lambda: I\left(c_{0}\right) \longrightarrow J\left(\eta_{0}\right)$ determined by Theorem 5.1. Then, $\Lambda$ is a strictly increasing function on $I\left(c_{0}\right)$.

Corollary 5.4. Consider $w:\left(\frac{L^{2}}{L^{2}-2 \pi^{2}},+\infty\right) \longrightarrow \mathbb{R}$, where $w$ is given by (5.8). Then $\frac{d w}{d c}>0$.

\subsection{Global well-posedness for the gSRLW equation}

In this section we study the initial value problem

$$
\left\{\begin{array}{l}
u_{t}-u_{x x t}+\left(u^{p+1}\right)_{x}-v_{x}=0 \\
v_{t}-u_{x}=0 \\
(u(0), v(0))=\left(u_{0}, v_{0}\right)
\end{array}\right.
$$

for $p \geq 1, p \in \mathbb{N}$, in the periodic setting. For $p=1$, a local and global wellposedness result for (5.9) in the periodic and continuous cases was obtained by Banquet in $[5]$ if $\left(u_{0}, v_{0}\right) \in H^{s}(\mathbb{R}) \times H^{s-1}(\mathbb{R})\left(\right.$ or in $\left.H_{\text {per }}^{s} \times H_{\text {per }}^{s-1}\right)$, with $s \geq 0$. For this reason we only consider $p \geq 2$. Write (5.9) in the form

$$
\left\{\begin{array}{l}
i u_{t}=\varphi\left(D_{x}\right)\left(u^{p+1}-v\right), \\
i v_{t}=-\psi\left(D_{x}\right) u \\
(u(0), v(0))=\left(u_{0}, v_{0}\right),
\end{array}\right.
$$

where $\widehat{\varphi\left(D_{x}\right)} u(\xi)=\frac{\xi}{1+|\xi|^{2}} \widehat{u}(\xi)=\varphi(\xi) \widehat{u}(\xi)$ and $\widehat{\psi\left(D_{x}\right)} u(\xi)=\xi \widehat{u}(\xi)=$ $\psi(\xi) \widehat{u}(\xi)$. Solving the linear problem

$$
\left\{\begin{array}{l}
i u_{t}=-\varphi\left(D_{x}\right)(v) \\
i v_{t}=-\psi\left(D_{x}\right) u \\
(u(0), v(0))=\left(u_{0}, v_{0}\right)
\end{array}\right.
$$

we get the solution $(u(t), v(t))=S(t)\left(u_{0}, v_{0}\right)$, where

$$
\left(\begin{array}{l}
\widehat{u}(t, \xi) \\
\widehat{v}(t, \xi)
\end{array}\right)=\left(\begin{array}{cc}
\cos (\alpha(\xi) t) & \frac{i}{\sqrt{1+|\xi|^{2}}} \sin (\alpha(\xi) t) \\
i \sqrt{1+|\xi|^{2}} \sin (\alpha(\xi) t) & \cos (\alpha(\xi) t)
\end{array}\right)\left(\begin{array}{l}
\widehat{u}_{0}(\xi) \\
\widehat{v}_{0}(\xi)
\end{array}\right)
$$


with $\alpha(\xi)=\frac{\xi}{\sqrt{1+|\xi|^{2}}}$. Then, (5.10) may be rewritten as the integral equation

$$
(u(x, t), v(x, t))=S(t)\left(u_{0}, v_{0}\right)-i \int_{0}^{t} S(t-\tau) G[(u, v)(x, \tau)] d \tau
$$

where $G$ is given by $G(u, v)=\left[\varphi\left(D_{x}\right)\left(u^{p+1}\right), 0\right]$. Using a fixed point argument and the fact that $H_{\text {per }}^{s}$ is a Banach algebra, it is easy to prove that the initial value problem (5.9) is locally well-posed in $H^{s} \times H^{s-1}$ for $s>1 / 2$. A global well-posedness result is obtained in $H_{\mathrm{per}}^{1} \times L_{\text {per }}^{2}$, from the fact that the norm $\|\cdot\|_{H_{\mathrm{per}}^{1} \times L_{\mathrm{per}}^{2}}$ is conserved by the flux of the system (5.9). Furthermore, using the implicit function theorem, it is obtained that the mapping data-solution associated to the generalized SRLW (gSRLW) equation is smooth.

Then we collect the previous conclusions in the following theorem. Define $X=H_{\text {per }}^{1} \times L_{\text {per }}^{2}$.

THEOREM 5.5. The Cauchy problem associated with the gSRLW equation (5.9) is globally well-posed in $X$; that is, if $\left(u_{0}, v_{0}\right) \in X$, there is a unique mild solution $(u, v) \in C([0, T] ; X)$ of (5.9), for all $T>0$. Moreover, the mapping data-solution associated to the gSRLW equation,

$$
\begin{aligned}
\Upsilon: X & \rightarrow C([0, T] ; X), \\
\left(u_{0}, v_{0}\right) & \mapsto \Upsilon\left(u_{0}, v_{0}\right)=\left(u_{u_{0}}, v_{v_{0}}\right),
\end{aligned}
$$

is smooth and we have that the following quantities

$$
\begin{aligned}
& V(u, v)=\frac{1}{2} \int_{0}^{L}\left(u_{x}^{2}+u^{2}+v^{2}\right) d x \\
& E(u, v)=\int_{0}^{L}\left(u v-\frac{1}{p+2} u^{p+2}\right) d x
\end{aligned}
$$

are conserved by the flow of the gSRLW equation.

\subsection{General stability criterion for the gSRLW equation}

In this section we establish a general criterion of orbital stability of traveling wave solutions for the gSRLW system (5.9). Let us consider $\left(\phi_{c}, \psi_{c}\right)$ satisfying

$$
\left\{\begin{array}{l}
c \phi_{c}^{\prime \prime}-c \phi_{c}+\phi_{c}^{p+1}-\psi_{c}=0 \\
-c \psi_{c}=\phi_{c}
\end{array}\right.
$$


Using the last system, we have that $\left(\phi_{c}, \psi_{c}\right)$ is a critical point for the functional $E(u, v)+c V(u, v)$, that is, $E^{\prime}\left(\phi_{c}, \psi_{c}\right)+c V^{\prime}\left(\phi_{c}, \psi_{c}\right)=0$. Now, define

$$
H_{c}=E^{\prime \prime}\left(\phi_{c}, \psi_{c}\right)+c V^{\prime \prime}\left(\phi_{c}, \psi_{c}\right)=\left[\begin{array}{cc}
c\left(1-\partial_{x}^{2}\right)-(p+1) \phi_{c}^{p} & 1 \\
1 & c
\end{array}\right] .
$$

Then the operator $H_{c}: D\left(H_{c}\right) \rightarrow L_{\text {per }}^{2} \times L_{\text {per }}^{2}$ is linear, closed, not bounded, and self-adjoint, defined on $H_{\text {per }}^{2} \times L_{\text {per }}^{2}$. Also it is easy to see that $H_{c}\left(\phi_{c}^{\prime}, \psi_{c}^{\prime}\right)=0$. Following the proof of the orbital stability of periodic traveling wave solutions for the SRLW equation given in Banquet [5] (see also Benjamin [6], Bona [7], Weinstein [23], and Grillakis, Shatah, and Strauss [10]) we obtain the following main conditions, which imply orbital stability in the case of the gSRLW equation:

$\left(C_{0}\right)$ there is a nontrivial smooth curve of periodic solutions for (5.9) of the form

$$
c \in I \subset \mathbb{R} \rightarrow\left(\phi_{c}, \psi_{c}\right) \in H_{\mathrm{per}}^{1}([0, L]) \times L^{2}([0, L]) ;
$$

$\left(C_{1}\right) \quad H_{c}$ has a unique negative eigenvalue and it is simple;

$\left(C_{2}\right)$ the eigenvalue zero is simple;

$\left(C_{3}\right) \quad \frac{d}{d c} \int_{-L}^{L}\left[\left(\phi_{c}^{\prime}\right)^{2}+\phi_{c}^{2}+\psi_{c}^{2}\right] d x>0$.

\subsection{Orbital stability of dnoidal waves for the mSRLW equation}

In this section we prove that the family of dnoidal waves established in Section 5.1 is orbitally stable by the periodic flow of the mSRLW. So, from Theorems 5.1 and 5.5 we only need to verify the conditions $\left(C_{1}\right),\left(C_{2}\right)$, and $\left(C_{3}\right)$ in (5.14).

5.4.1. Proof of conditions $\left(C_{1}\right)$ and $\left(C_{2}\right)$. We consider the linear operator $H_{c}$ given in (5.13), with $p=2$. Then from (5.12) we get that $H_{c}\left(\phi_{c}^{\prime}, \psi_{c}^{\prime}\right)=0$. Therefore zero is an eigenvalue with associated eigenfunction $\left(\phi_{c}^{\prime}, \psi_{c}^{\prime}\right)$. The theory of compact self-adjoint operators implies that $H_{c}$ has a compact resolvent and so the spectrum of $H_{c}$ is a countable infinity set of eigenvalues $\left\{\lambda_{n}\right\}_{n=0}^{\infty}$ with $\lambda_{0} \leq \lambda_{1} \leq \lambda_{2} \leq \cdots$, where $\lambda_{n} \rightarrow \infty$ as $n \rightarrow \infty$.

Next we consider the following operator of Schrödinger type

$$
\mathcal{L}=-c \frac{d^{2}}{d x^{2}}+\frac{c^{2}-1}{c}-3 \phi_{c}^{2} .
$$


Following the same lines of Angulo Pava [1, Theorem 3.1] (see also Angulo Pava [2]), we get the next result.

Proposition 5.6. Let $\phi_{c}$ be the periodic wave solution given by Theorem 5.1, with $c>\frac{L}{\sqrt{L^{2}-2 \pi^{2}}}$ and $L>2 \pi$. Then, the operator $\mathcal{L}$ defined in (5.15) with domain $H_{\mathrm{per}}^{2}([0, L])$ has its first two eigenvalues simple with zero being the second one. Moreover, the remainder of the spectrum is constituted by a discrete set of eigenvalues which converge to $+\infty$.

Now, we calculate the quadratic form associated to the operator $H_{c}$, which is given for $(f, g)$ in a dense subset of $X:=H_{\mathrm{per}}^{1}([0, L]) \times L_{\mathrm{per}}^{2}([0, L])$ by

$$
\begin{aligned}
\langle H(f, g),(f, g)\rangle & =\int\left(-c f^{\prime \prime} f+c f^{2}-\phi_{c} f^{2}+c g^{2}+2 f g\right) d x \\
& =\langle\mathcal{L} f, f\rangle_{L_{\mathrm{per}}^{2}}+\frac{1}{c}\|c g+f\|_{L_{\mathrm{per}}^{2}}^{2} .
\end{aligned}
$$

Here we denote $\langle(f, g),(h, j)\rangle:=\int(f h+g j) d x$. The next theorem shows us the behavior of the first two eigenvalues related to the operator $H_{c}$.

THEOREM 5.7. Let $\left(\phi_{c}, \psi_{c}\right)$ be the traveling wave solution given by Theorem 5.1, and let $c>\frac{L}{\sqrt{L^{2}-2 \pi^{2}}}$. Then, the operator $H_{c}$ with domain $H_{\mathrm{per}}^{2}([0, L]) \times L_{\mathrm{per}}^{2}([0, L])$ in $L_{\mathrm{per}}^{2}([0, L]) \times L_{\mathrm{per}}^{2}([0, L])$ has its first three eigenvalues simple. The eigenvalue zero is the second one with associated eigenfunction $\left(\phi_{c}^{\prime}, \psi_{c}^{\prime}\right)$. Moreover, the remainder of the spectrum is constituted by a discrete set of eigenvalues.

Proof. Using system (5.12), we have that $H_{c}\left(\phi_{c}^{\prime}, \psi_{c}^{\prime}\right)=\overrightarrow{0}$. Thus zero is an eigenvalue of $H_{c}$. Now, assume that $(f, g) \neq \overrightarrow{0}$ is such that $H_{c}(f, g)=\overrightarrow{0}$. Then, $\mathcal{L}_{0} f+g=0$ and $c g+f=0$, where $\mathcal{L}_{0}=c\left(1-\partial_{x}^{2}\right)-3 \phi_{c}^{2}$. After some algebra we get that $\mathcal{L} f=0$. Therefore, there exists $a \in \mathbb{R}-\{0\}$ such that $f=a \phi_{c}^{\prime}$ and consequently that $g=-\frac{a}{c} \phi_{c}^{\prime}=a \psi_{c}^{\prime}$. This shows that zero is a simple eigenvalue of $H_{c}$ with associated eigenfunction $\left(\phi_{c}^{\prime}, \psi_{c}^{\prime}\right)$. Let $\kappa$ be the unique negative eigenvalue of $\mathcal{L}$ with eigenfunction $\nu$. Since $\langle\mathcal{L} f, f\rangle_{L_{\mathrm{per}}^{2}}$ assumes negative values, from (5.16) we have that $\left\langle H_{c}(f, g),(f, g)\right\rangle$ assumes negative values. Indeed, consider $\vec{\psi}=(-c \nu, \nu)$. Then

$$
\left\langle H_{c} \vec{\psi}, \vec{\psi}\right\rangle=c^{2}\langle\mathcal{L} \nu, \nu\rangle_{L_{\mathrm{per}}^{2}}+\frac{1}{c}\|c \nu-c \nu\|_{L_{\mathrm{per}}^{2}}^{2}=\kappa c^{2}\|\nu\|_{L_{\mathrm{per}}^{2}}^{2}<0
$$


Moreover, the smallest eigenvalue associated to $H_{c}$, say, $\sigma_{1}$, is negative. We will show that the next eigenvalue of $H_{c}$ is $\sigma_{2}=0$ (which is already known to be simple) and consequently that the third eigenvalue $\sigma_{3}$ is positive. For this purpose we use the min-max characterization of eigenvalues (see Reed and Simon [19]). Namely, we have

$$
\sigma_{2}=\max _{(f, g) \in X} \min _{(h, j) \in X_{(f, g)}} \frac{\left\langle H_{c}(h, j),(h, j)\right\rangle}{\|(h, j)\|_{X}},
$$

where $X_{(f, g)}=\left\{(h, j) \in X-\{0\}:(h, f)_{1}+\langle j, g\rangle_{L_{\text {per }}^{2}}=0\right\}$. Here $(\cdot, \cdot)_{1}$ denotes the inner product in $H_{\text {per }}^{1}([0, L])$. If we choose $f=\nu$ and $g=0$, we get immediately from Proposition 5.6 that

$$
\sigma_{2} \geq \min _{(h, j) \in X_{(\nu, 0)}} \frac{\left\langle H_{c}(h, j),(h, j)\right\rangle}{\|(h, j)\|_{X}} \geq 0,
$$

and therefore $\sigma_{2}=0$. Since 0 is simple, we obtain that $\sigma_{3}>0$.

5.4.2. Proof of condition $\left(C_{3}\right)$. In the following analysis we take advantage of the formula (5.4).

Proposition 5.8. Let $L>\sqrt{2} \pi$, and let $c>\frac{L}{\sqrt{L^{2}-2 \pi^{2}}}$. Then for $V(c)=$ $\int_{0}^{L}\left(\phi_{c}^{2}+\phi_{c}^{\prime 2}+\psi_{c}^{2}\right) d x$, we have $\frac{d}{d c} V(c)>0$.

Proof. Since $c \psi_{c}=-\phi_{c}$, and using the Parseval identity, we obtain

$$
\frac{d}{d c} V(c)=L \frac{d}{d c}\left\|\left(\frac{c^{2}+1}{c^{2}}+|\cdot|^{2}\right)^{1 / 2} \widehat{\phi}_{c}\right\|_{l^{2}}^{2} .
$$

But we have that $\widehat{\phi}_{c}(n)=\sqrt{\frac{c}{w(c)}} \widehat{\phi_{w(c)}}(n)=\frac{\sqrt{2 c} \pi}{L} \operatorname{sech}\left(\sqrt{\frac{w^{2}(c)}{w^{2}(c)-1}} \frac{\pi n}{2 L}\right)$ and consequently

$$
V(c)=\frac{2 \pi^{2}}{L^{2}} \sum_{n=-\infty}^{\infty}\left(\frac{c^{2}+1}{c}+c|n|^{2}\right) \operatorname{sech}^{2}\left(\frac{\pi n}{2 L} \sqrt{\frac{w^{2}}{w^{2}-1}}\right) .
$$

Hence,

$$
\begin{aligned}
\frac{d}{d c} V(c)= & \frac{D}{\left(w^{2}-1\right)^{3 / 2}} \frac{d w}{d c} \\
& \cdot \sum_{n=-\infty}^{\infty}\left(\frac{c^{2}+1}{c^{2}}+c|n|^{2}\right) n \operatorname{sech}^{2}\left(\frac{\pi n}{2 L} \sqrt{\frac{w^{2}}{w^{2}-1}}\right)
\end{aligned}
$$




$$
\begin{aligned}
& \cdot \tanh \left(\frac{\pi n}{2 L} \sqrt{\frac{w^{2}}{w^{2}-1}}\right) \\
& +\frac{2 \pi^{2}}{L^{2}} \sum_{n=-\infty}^{\infty}\left(\frac{c^{2}-1}{c^{2}}+|n|^{2}\right) \operatorname{sech}^{2}\left(\frac{\pi n}{2 L} \sqrt{\frac{w^{2}}{w^{2}-1}}\right),
\end{aligned}
$$

where the constant $D=D(c, L)>0$. Since the sequence $\left\{n \tanh \left(\frac{\pi n}{2 L} \sqrt{\frac{w^{2}}{w^{2}-1}}\right)\right\}_{n}$ is positive, using Corollary 5.4 we get that $\frac{d}{d c} V(c)>0$.

From the analysis above we have the following theorem of stability.

TheOREM 5.9 (Stability of dnoidal waves for the mSRLW equation). Let $L>\sqrt{2} \pi$, and let $c>\frac{L}{\sqrt{L^{2}-2 \pi^{2}}}$. Let $\left(\phi_{c}, \psi_{c}\right)$ be the traveling wave solution given by Theorem 5.1. Then the $\Omega_{\left(\phi_{c}, \psi_{c}\right)}$-orbit is stable by the periodic flow of the mSRLW equation (5.1).

\subsection{Linear instability of cnoidal waves for the mSRLW equation}

Here we apply the results established in the Section 4 to conclude the linear instability of cnoidal waves for the mSRLW equation. Assuming $c \neq 0$, from (5.3) we obtain the differential equation in quadrature form,

$$
\left[\phi_{c}^{\prime}\right]^{2}=\frac{1}{2 c}\left[-\phi_{c}^{4}+\frac{2\left(c^{2}-1\right)}{c} \phi_{c}^{2}+4 D_{\phi_{c}}\right],
$$

where $D_{\phi_{c}}$ is a nonzero integration constant. The periodic solutions related to (5.3) can be obtained from the specific form of the roots associated to the polynomial $Q(t)=-t^{4}+\frac{2(c-1)}{c} t^{2}+4 D_{\phi_{c}}$. If we suppose that $Q$ has two symmetric real roots, $-b<0<b$ and a pure imaginary root $i a$, we obtain for $c>1$ the formula (see Angulo Pava [1], [2])

$$
\varphi_{c}(x)=b \operatorname{cn}\left(\frac{\beta}{\sqrt{c}} x ; k\right)
$$

which is a periodic sign-changing solution for (5.3). Here, we have $k^{2}=$ $\frac{b^{2}}{a^{2}+b^{2}}, b^{2}-a^{2}=\frac{2\left(c^{2}-1\right)}{c}$, and $\beta=\sqrt{\frac{a^{2}+b^{2}}{2}}$. We note that for $c>1$, we get that $b^{2}>\frac{2\left(c^{2}-1\right)}{c}$. Since $k^{2}=\frac{b^{2}}{j(b, c)}$ for $j(b, c)=2 b^{2}-\frac{2\left(c^{2}-1\right)}{c}$, we must have $k^{2} \in(1 / 2,1)$. Next, since the cnoidal has real fundamental period equal to 
$4 K$, we obtain that the fundamental period for $\varphi_{c}$ can be seen as a function of $b$,

$$
T_{\varphi_{c}}(b)=\frac{4 \sqrt{c}}{\sqrt{\frac{1}{2} j(b, c)}} K(k(b)) .
$$

Then, applying a similar argument as in [1, Theorem 2.3], we can deduce from the implicit function theorem the following result.

THEOREM 5.10. Let $L>0$ be fixed, and let $k^{2} \in(1 / 2,1)$ satisfying $L^{2}>$ $16 K^{2}(k)\left(2 k^{2}-1\right)$. Then we have the following.

(i) For every $c>1$ there is a unique $b=b(c) \in\left(\sqrt{\frac{2\left(c^{2}-1\right)}{c}},+\infty\right)$ such that the map $c \in(1,+\infty) \mapsto b(c)$ is a strictly increasing smooth function and $L=\frac{4 \sqrt{c}}{\sqrt{\frac{1}{2} j(b(c), c)}} K(k)$. The modulus $k=k(c)$ is given by $k^{2}(c)=\frac{b^{2}(c)}{j(b(c), c)}$ and $\frac{d k}{d c}>0$.

(ii) For every $c>1$ and $h(c) \equiv \frac{1}{2 c} j(b(c), c)$, the wave

$$
\left(\phi_{c}(x), \psi_{c}(x)\right)=\left(b \operatorname{cn}(\sqrt{h(c)} \cdot x ; k),-\frac{b}{c} \operatorname{cn}(\sqrt{h(c)} \cdot x ; k)\right)
$$

has fundamental period $L$ and satisfies equation (5.2). Moreover, the mapping $c \in(1,+\infty) \mapsto\left(\phi_{c}, \psi_{c}\right) \in H_{\mathrm{per}}^{n}([0, L]) \times H_{\mathrm{per}}^{n}([0, L])$ is a smooth function for all $n \in \mathbb{N}$.

Next, for $\mathcal{L}_{0}$ defined in $(1.10)$ with $f(u)=u^{3}$, we consider the eigenvalue problem in $H_{\text {per }}^{2}([0, L])$

$$
\left\{\begin{array}{l}
\mathcal{L}_{0} \psi=\eta \psi, \\
\psi(0)=\psi(L), \quad \psi^{\prime}(0)=\psi^{\prime}(L),
\end{array}\right.
$$

which immediately implies the existence of an enumerable set of eigenvalues $\left\{\eta_{i}\right\}_{i \geqq 0}$. From (5.17) and the transformation $\Phi(x)=\psi(\sqrt{c} x / \beta)$, we obtain the eigenvalue problem

$$
\begin{gathered}
L \Phi:=-\Phi^{\prime \prime}+6 k^{2} \operatorname{sn}^{2}(x ; k) \Phi=\theta \Phi, \\
\Phi(0)=\Phi(4 K(k)) \Phi^{\prime}(0)=\Phi^{\prime}(4 K(k)) .
\end{gathered}
$$

Here $\theta$ will be an eigenvalue satisfying

$$
\theta=\frac{1}{\beta^{2}}\left[3 b^{2}-\frac{c^{2}-1}{c}+c \eta\right] .
$$


Now, it is known that for (5.18) the set of eigenvalues $\left\{\theta_{i}\right\}_{i \geqq 0}$ has the distribution

$$
\theta_{0}<\theta_{1}<\theta_{2}<\theta_{3}<\theta_{4}<\theta_{5} \leqq \theta_{6}<\cdots,
$$

which means that the first five eigenvalues are simple and that all the other eigenvalues have multiplicity 2 (see Magnus and Winkler [17]). Since the exact value of these five eigenvalues, as well as the associated eigenfunctions, will be useful for all subsequent calculations, we have the following:

$$
\begin{array}{lll}
\theta_{0}=2\left[1+k^{2}-r(k)\right] ; & \psi_{0}(x)=1-\left(1+k^{2}-r(k)\right) \operatorname{sn}^{2}(x), \\
\theta_{1}=1+k^{2} ; & & \psi_{1}(x)=\partial_{x} \operatorname{sn}(x)=\operatorname{cn}(x) \operatorname{dn}(x), \\
\theta_{2}=1+4 k^{2} ; & & \psi_{2}(x)=\partial_{x} \operatorname{cn}(x)=-\operatorname{sn}(x) \operatorname{dn}(x), \\
\theta_{3}=4+k^{2} ; & \psi_{3}(x)=\partial_{x} \operatorname{dn}(x)=-k^{2} \operatorname{sn}(x) \operatorname{cn}(x), \\
\theta_{4}=2\left[1+k^{2}+r(k)\right] ; & \psi_{4}(x)=1-\left(1+k^{2}+r(k)\right) \operatorname{sn}^{2}(x),
\end{array}
$$

where $r(k)=\sqrt{1-k^{2}+k^{4}}$. Moreover, the following basic computation shows that for $j \neq 0$ and $j \neq 4$, we have that the associated eigenfunction $\Phi_{j}$ has zero mean. Indeed, $L \Phi_{j}=\theta_{j} \Phi_{j}$ implies that

$$
\theta_{j}\left\langle\Phi_{j}, 1\right\rangle=6\left\langle k^{2} \mathrm{sn}^{2}, \Phi_{j}\right\rangle=6\left\langle\Phi_{j}, \Phi_{4}\right\rangle+2\left[1+k^{2}-r(k)\right]\left\langle\Phi_{j}, 1\right\rangle .
$$

Since $\theta_{j}>\theta_{0}$, we obtain $\left\langle\Phi_{j}, 1\right\rangle=0$.

Now, using the same steps as in [1, Proof of Theorem 2.3], we obtain that $n^{-}\left(\mathcal{L}_{0}\right)=2$ and that $\operatorname{ker}\left(\mathcal{L}_{0}\right)=\left[\frac{d}{d x} \phi_{c}\right]$ (from (5.19), the eigenvalues $\theta_{0}, \theta_{1}$, and $\theta_{2}$ determine $\eta_{0}, \eta_{1}$, and $\eta_{2}=0$, respectively; moreover, $\eta_{0}<\eta_{1}<\eta_{2}=$ $0)$. Furthermore, since $\left\langle\Psi_{1}, 1\right\rangle=\int_{0}^{4 K(k)} \partial_{x} s n(x ; k) d x=0$, we obtain that the eigenvalue $\eta_{1}$ belongs to the negative spectrum of $Q \mathcal{L}_{0}$, so we have that $1 \leq n^{-}\left(Q \mathcal{L}_{0}\right) \leq 2$. Next, we obtain the sign of $I(c)$ given in (4.1). From Theorem 5.10 we obtain that

$$
\frac{d V(c)}{d c}=2 \frac{d}{d c}\left\{\frac{b^{2}}{\alpha} \int_{0}^{K}\left[\frac{c^{2}+1}{c^{2}} \operatorname{cn}^{2}(x ; k)+\alpha^{2} \operatorname{sn}^{2}(x ; k) \operatorname{dn}^{2}(x ; k)\right] d x\right\},
$$

with $\alpha=4 K / L$. Moreover, for all $L>0$ we can write $b^{2}=\frac{32 c k^{2} K^{2}(k)}{L^{2}}$ and the wave speed $c>1$ by the expression

$$
c^{2}=\frac{L^{2}}{L^{2}-16 K^{2}(k)\left(2 k^{2}-1\right)}, \quad k^{2} \in(1 / 2,1) .
$$

Now, from Byrd and Friedman [9], we have

$$
\int_{0}^{K} \operatorname{cn}^{2}(x ; k) d x=\frac{1}{k^{2}}\left[E(k)-\left(1-k^{2}\right) K(k)\right],
$$


with $E=E(k)$ the complete elliptic integral of the second kind, and

$$
\alpha^{2} \int_{0}^{K} \operatorname{sn}^{2}(x ; k) \mathrm{dn}^{2}(x ; k) d x=\frac{16 K(k)^{2}}{3 k^{2} L^{2}}\left[\left(2 k^{2}-1\right) E(k)+\left(1-k^{2}\right) K(k)\right] .
$$

Therefore,

$$
\begin{aligned}
\frac{d V(c)}{d c}= & 16 \frac{d}{d c}\left\{\frac{c^{2}+1}{c} \frac{K}{L}\left[E-\left(1-k^{2}\right) K\right]\right. \\
& \left.+\frac{16 c K^{3}}{3 L^{3}}\left[\left(2 k^{2}-1\right) E+\left(1-k^{2}\right) K\right]\right\} \\
= & 16 \frac{d}{d c}\left\{\frac{c^{2}+1}{c} \frac{D_{1}(k)}{L}+\frac{16 c D_{2}(k)}{3 L^{3}}\right\} .
\end{aligned}
$$

Since $c \in(1,+\infty) \mapsto \frac{c^{2}+1}{c}, k \in(0,1) \mapsto D_{1}(k), k \in(0,1) \mapsto D_{2}(k)$ are positive strictly increasing functions and $\frac{d k}{d c}>0$ in $\left(\frac{\sqrt{2}}{2}, 1\right)$, we have from (5.20) that $\frac{d V(c)}{d c}>0$ and then $I(c)<0$. Using a very similar argument as in [4], we get that, for $k \in\left(\sqrt{2} / 2, k^{*}\right)$, we have $n^{-}\left(Q \mathcal{L}_{0}\right)=1$ and for $k \in\left(k^{*}, 1\right)$, we have $n^{-}\left(Q \mathcal{L}_{0}\right)=2$, where $k^{*} \sim 0.909$. Moreover, $\operatorname{ker}\left(Q \mathcal{L}_{0}\right)=\left[\frac{d}{d x} \phi_{c}\right]$.

The following result is obtained from Theorem 1.1.

THEOREM 5.11 (Linear instability of cnoidal waves for the mSRLW equation). The solution $\left(\phi_{c}, \psi_{c}\right)$, where $c \psi_{c}=-\phi_{c}$ and $\phi_{c}$ is given in (5.17), is linearly unstable for the $M S R L W$ equation (5.1), provided that the wave speed $c \in\left(c^{*},+\infty\right)$, where

$$
c^{* 2}=\frac{L^{2}}{L^{2}-16 K^{2}\left(k^{*}\right)\left(2 k^{* 2}-1\right)} .
$$

REMARK 5.12.

(1) We can determine numerically the value of $c^{* 2} \approx \frac{L^{2}}{L^{2}-56,277}$, and so our minimal period $L$ must satisfy a priori the lower bounded $L^{2}>56,277$.

(2) Since $\operatorname{ker}\left(Q \mathcal{L}_{0}\right)=\operatorname{span}\left\{\frac{d}{d x} \varphi_{c}\right\}$ and $n^{-}\left(Q \mathcal{L}_{0}\right)=1$ for $k \in\left(\sqrt{2} / 2, k^{*}\right)$, we obtain from Grillakis, Shatah, and Strauss [10] that the orbit $\Omega_{\varphi_{c}}$ is stable in $H_{\text {per }}^{1}([0, L]) \cap \mathbb{V}$ for $c \in\left(1, c^{*}\right)$.

\subsection{Nonlinear instability of cnoidal waves for the mSRLW equation}

In this section we establish the nonlinear instability of the linearly unstable cnoidal waves for the mSRLW equation determined in Theorem 5.11. 
The following theorem is the link for obtaining nonlinear instability from a linear instability result.

Theorem 5.13. Let $Y$ be a Banach space, and let $\Omega \subset Y$ be an open set containing 0 . Suppose that $T: \Omega \rightarrow Y$ has $T(0)=0$, and for some $p>1$ and continuous linear operator $\mathcal{L}$ with spectral radius $r(\mathcal{L})>1$, we have that $\|T(x)-\mathcal{L} x\|_{Y}=O\left(\|x\|_{Y}^{p}\right)$ as $x \rightarrow 0$. Then 0 is unstable as a fixed point of $T$.

Proof. See Henry, Perez, and Wreszinski [12, Theorem 2].

REMARK 5.14. In Theorem 5.13, 0 is unstable as a fixed point of $T$ if there is $\varepsilon_{0}>0$ such that for all $B(0 ; \eta)$ and arbitrarily large $N_{0} \in \mathbb{N}$, there is a $N>N_{0}$ and $x \in B(0 ; \eta)$ such that $\left\|T^{N}(x)\right\|_{Y} \geq \varepsilon_{0}$.

By using Taylor's theorem, Theorem 5.13 implies immediately the following result.

Corollary 5.15. Let $S: \Omega \subset Y \rightarrow Y$ be a $C^{2}$ map defined in an open neighborhood of a fixed point $\varphi$ of $S$. If there is an element $\mu \in \sigma\left(S^{\prime}(\varphi)\right)$ with $|\mu|>1$, then $\varphi$ is an unstable fixed point of $S$.

THEOREM 5.16. The cnoidal profile solution $\left(\phi_{c}, \psi_{c}\right)$, where $c \psi_{c}=-\phi_{c}$ and $\phi_{c}$ is given in (5.17), is nonlinearly unstable for the mSRLW equation (5.1), provided that the wave speed $c \in\left(c^{*},+\infty\right)$ with $c^{*}$ defined as in (5.21).

Proof. In system (5.1), we replace $(u(x, t), v(x, t))$ by $(u(x+c t, t), v(x+$ $c t, t)$ ) and then we obtain

$$
\left\{\begin{array}{l}
u_{t}-c u_{x}+3 u^{2} u_{x}+c u_{x x x}-u_{x x t}-v_{x}=0 \\
v_{t}-c v_{x}-u_{x}=0
\end{array}\right.
$$

Then $\left(\phi_{c}, \psi_{c}\right)$ is an equilibrium solution for equation (5.22). Defining $G(u, v)=\frac{1}{c}[E(u, v)+c V(u, v)]$, where $E$ and $F$ are defined as in (5.11), we have that equation $(5.22)$ can be written as

$$
\left(\left(1-\partial_{x}^{2}\right) u, v\right)_{t}=J G^{\prime}(u, v),
$$

where $J=\left[\begin{array}{cc}c \partial_{x} & 0 \\ 0 & c \partial_{x}\end{array}\right]$. Moreover, from (5.23) we see that the linearized equation at equilibrium point $\left(\phi_{c}, \psi_{c}\right)$ is $\left(\left(1-\partial_{x}^{2}\right) w, z\right)_{t}=J H_{0}(w, z)$ (see (1.6)), where $H_{0}$ is the linear self-adjoint operator defined by

$$
H_{0}=\left[\begin{array}{cc}
1-\partial_{x x}-\frac{1}{c} f^{\prime}\left(\phi_{c}\right) & \frac{1}{c} \\
\frac{1}{c} & 1
\end{array}\right]
$$


Let us define $S: X \rightarrow X$ as $S\left(u_{0}, v_{0}\right)=\left(u_{u_{0}}(1), v_{v_{0}}(1)\right)$, where $\left(u_{u_{0}}(t), v_{v_{0}}(t)\right)$ is the solution of $(5.22)$ with initial data $(u(x, 0), v(x, 0))=$ $\left(u_{0}(x), v_{0}(x)\right)$. If one considers $\Upsilon_{c}: X \rightarrow C([0, T] ; X)$ the mapping datasolution related to equation (5.22), we see from Theorem 5.5 that $\Upsilon_{c}$ is smooth. Furthermore, $S\left(\phi_{c}, \psi_{c}\right)=\left(\phi_{c}, \psi_{c}\right)$ and $S$ is a $C^{2}$ map defined on a neighborhood of $\left(\phi_{c}, \psi_{c}\right)$ (this last fact follows since translation in $x$ is a linear continuous map in $X)$. Moreover, for $(g, h) \in X$ we have $S^{\prime}\left(\phi_{c}, \psi_{c}\right)(g, h)=\left(w_{g}(1), z_{h}(1)\right)$, where $\left(w_{g}(1), z_{h}(1)\right)$ is the solution of the linear initial value problem

$$
\left\{\begin{array}{l}
\left(\left(1-\partial_{x}^{2}\right) w, z\right)_{t}=J H_{0}(w, z), \\
(w, z)(0)=(g, h)
\end{array}\right.
$$

evaluated in $t=1$. Then, from arguments established in Sections 4 and 5.5, we deduce that there is $\lambda>0$ and $\left(w_{0}, z_{0}\right) \in X-\{0\}$ such that $J H_{0}\left(w_{0}, z_{0}\right)=$ $\lambda\left(\left(1-\partial_{x}^{2}\right) w_{0}, z_{0}\right)$. Hence for $\left(w_{w_{0}}(t), z_{z_{0}}(t)\right)=e^{\lambda t}\left(w_{0}, z_{0}\right)$ and $\mu:=e^{\lambda}$, we obtain $S^{\prime}\left(\phi_{c}, \psi_{c}\right)\left(w_{0}, z_{0}\right)=\mu\left(w_{0}, z_{0}\right)$. Therefore $\mu \in \sigma\left(S^{\prime}\left(\phi_{c}, \psi_{c}\right)\right)$, and from Corollary 5.15 we obtain the nonlinear instability in $X$ of the cnoidal solution $\left(\phi_{c}, \psi_{c}\right)$, provided that $c \in\left(c^{*},+\infty\right)$.

Acknowledgments. J. Angulo Pava was partially supported by a CNPq grant (Brazil) and by São Paulo State Research Foundation (FAPESP) project 2012/50521-4. The authors would like to thank the anonymous referees for constructive suggestions concerning the presentation of this work.

\section{REFERENCES}

[1] J. Angulo Pava, Nonlinear stability of periodic traveling wave solutions to the Schrödinger and the modified Korteweg-de Vries equations, J. Differential Equations 235 (2007), 1-30. MR 2309564. DOI 10.1016/j.jde.2007.01.003.

[2] - Nonlinear Dispersive Equations: Existence and Stability of Solitary and Periodic Travelling Wave Solutions, Math. Surveys Monogr. 156, Amer. Math. Soc., Providence, 2009. MR 2567568. DOI 10.1090/surv/156.

[3] J. Angulo Pava, C. Banquet, and M. Scialom, Stability for the modified and fourthorder Benjamin-Bona-Mahony equations, Discrete Contin. Dyn. Syst. 30 (2011), 851871. MR 2784624. DOI 10.3934/dcds.2011.30.851.

[4] J. Angulo Pava and F. Natali, Instability of periodic waves for nonlinear dispersive models, preprint, 2012.

[5] C. Banquet, The symmetric regularized-long-wave equation: Well-posedness and nonlinear stability, Phys. D 241 (2012), 125-133.

[6] T. B. Benjamin, The stability of solitary waves, Proc. Roy. Soc. Lond. Ser. A 338 (1972), 153-183. MR 0338584. 
[7] J. Bona, On the stability theory of solitary waves, Proc. Roy. Soc. Lond. Ser. A 344 (1975), 363-374. MR 0386438.

[8] J. Boussinesq, Théorie des ondes et des remous qui se propagent le long d'un canal rectangulaire horizontal, en communiquant au liquide contenu dans ce canal des vitesses sensiblement pareilles de la surface au fond, J. Math. Pures Appl. Ser. 2 17 (1872), 55-108.

[9] P. F. Byrd and M. D. Friedman, Handbook of Elliptic Integrals for Engineers and Scientists, 2nd ed., revised, Grundlehren Math. Wiss. 67, Springer, New York, 1971. MR 0277773.

[10] M. Grillakis, J. Shatah, and W. Strauss, Stability theory of solitary waves in the presence of symmetry, I, J. Funct. Anal. 74 (1987), 160-197. MR 0901236. DOI 10.1016/0022-1236(87)90044-9.

[11] - Stability theory of solitary waves in the presence of symmetry, II, J. Funct. Anal. 94 (1990), 308-348. MR 1081647. DOI 10.1016/0022-1236(90)90016-E.

[12] D. B. Henry, J. F. Perez, and W. F. Wreszinski, Stability theory for solitary-wave solutions of scalar field equations, Comm. Math. Phys. 85 (1982), 351-361. MR 0678151.

[13] P. D. Hislop and I. M. Sigal, Introduction to Spectral Theory: With Application to Schrödinger Operators, Appl. Math. Sci. 113, Springer, New York, 1996. MR 1361167. DOI 10.1007/978-1-4612-0741-2.

[14] R. J. Iorio. Jr. and V. Iorio, Fourier Analysis and Partial Differential Equations, Cambridge Stud. Adv. Math. 70, Cambridge University Press, Cambridge, 2001. MR 1826392. DOI 10.1017/CBO9780511623745.

[15] T. Kato, Perturbation Theory for Linear Operators, 2nd ed., Grundlehren Math. Wiss. 132, Springer, Berlin, 1976. MR 0407617.

[16] Z. Lin, Instability of nonlinear dispersive solitary waves, J. Funct. Anal. 255 (2008), 1191-1224. MR 2455496. DOI 10.1016/j.jfa.2008.06.003.

[17] W. Magnus and S. Winkler, Hill's Equation, Interscience Tracts in Pure Appl. Math. 20, Wiley, New York, 1966. MR 0197830.

[18] E. Oberhettinger, Fourier Expansions: A Collection of Formulas, Academic Press, New York, 1973. MR 0352886.

[19] S. Reed and B. Simon, Methods of Modern Mathematical Physics, IV: Analysis of Operators, Academic Press, New York, 1978. MR 0493421.

[20] C. Seyler and D. Fenstermacher, A symmetric regularized-long-wave equation, Phys. Fluids 27 (1984), 4-7.

[21] E. M. Stein and G. Weiss, Introduction to Fourier Analysis on Euclidean Spaces, Princeton Math. Ser. 32, Princeton University Press, Princeton, 1971. MR 0304972.

[22] E. Vock and W. Hunziker, Stability of Schrödinger eigenvalue problems, Comm. Math. Phys. 83 (1982), 281-302. MR 0649163.

[23] M. I. Weinstein, Lyapunov stability of ground states of nonlinear dispersive evolution equations, Comm. Pure Appl. Math. 39 (1986), 51-67. MR 0820338. DOI 10.1002/cpa.3160390103. 
Jaime Angulo Pava

Department of Mathematics

Instituto de Matemática e Estatística

Universidade de São Paulo

CEP 05508-090, São Paulo, SP

Brazil

angulo@ime.usp.br

Carlos Alberto Banquet Brango

Departamento de Matemáticas y Estadística

Universidad de Córdoba

Código Postal 230002

Colombia

cbanquet@correo.unicordoba.edu. co 